\title{
Wet Environment Effects for Ethanol and Water Adsorption on Anatase $\mathrm{TiO}_{2}(101)$ Surfaces
}

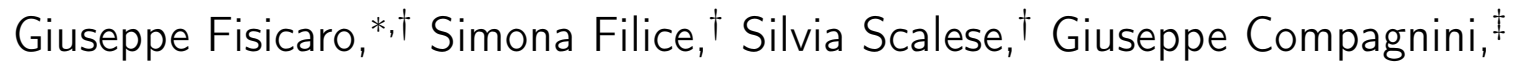 \\ Riccardo Reitano, " Luigi Genovese, ${ }^{\S}$ Stefan Goedecker,"l loannis Deretzis, ${ }^{* \dagger}$ and \\ Antonino La Magna ${ }^{\dagger}$ \\ $\dagger$ †onsiglio Nazionale delle Ricerche, Istituto per la Microelettronica e Microsistemi \\ (CNR-IMM), Z.I. VIII Strada 5, I-95121 Catania, Italy \\ $\ddagger$ Dipartimento di Scienze Chimiche, Università di Catania, Viale A. Doria 6, I-95125 \\ Catania, Italy \\ IDipartimento di Fisica e Astronomia, via S. Sofia 64, I-95123 Catania, Italy \\ $\S$ Laboratoire de simulation atomistique (L_Sim), SP2M, INAC, CEA-UJF, F-38054 \\ Grenoble, France \\ ||Department of Physics, University of Basel, Klingelbergstrasse 82, CH-4056 Basel, \\ Switzerland \\ E-mail: Giuseppe.Fisicaro@imm.cnr.it; loannis.Deretzis@imm.cnr.it
}

\begin{abstract}
Titanium dioxide exhibits superior photocatalytic properties, mainly occurring in liquid environments through molecular adsorptions and dissociations at the solid/liquid interface. The presence of these wet environments is often neglected when performing ab initio calculations for the interaction between the adsorbed molecules and the $\mathrm{TiO}_{2}$
\end{abstract}


surface. In this study we consider two solvents, i.e. water and ethanol, and show that the proper inclusion of the wet environment in the methodological scheme is fundamental for obtaining reliable results. Our calculations are based on structure predictions at a density functional theory level for molecules interacting with the perfect and defective anatase (1 0101$)$ surface under both vacuum and wet conditions. A soft-sphere implicit solvation model is used to describe the polar character of the two solvents. As a result, we find that surface oxygen vacancies become energetically favorable with respect to subsurface vacancies at the solid/liquid interface. This aspect is confirmed by ab initio molecular dynamics simulations with explicit water molecules. Ethanol molecules are able to strongly passivate these vacancies, whereas water molecules only weakly interact with the (101) surface, allowing the coexistence of surface vacancy defects and adsorbed species. Infrared and photoluminescence spectra of anatase nanoparticles exposing predominantly (101) surfaces dispersed in water and ethanol support the predicted molecular-surface interactions, validating the whole computational paradigm. The combined analysis allows for a better interpretation of $\mathrm{TiO}_{2}$ processes in wet environments based on improved computational models with implicit solvation features.

\section{Introduction}

Titanium dioxide $\left(\mathrm{TiO}_{2}\right)$ has received great attention for its potential role in clean energy and in carbon-free production of hydrogen. ${ }^{1}$ Photoassisted water splitting, ${ }^{2}$ photocatalysis, ${ }^{3-5}$ solar energy conversion ${ }^{6}$ and photo-electrocatalytic environmental cleanup ${ }^{7}$ are some relevant examples. Its anatase phase detains superior oxidation and photocatalytic properties with respect to the rutile polymorph. Furthermore, due to its lower surface energies it is more stable for particles below $\sim 14 \mathrm{~nm}$ in size, ${ }^{1,8}$ which are relevant for catalytic applications. These anatase nanocrystals mainly expose two low index surfaces, i.e. (001) and (10 1). The anatase $\mathrm{TiO}_{2}\left(\begin{array}{ll}0 & 0\end{array}\right)$ surface $\left(\mathrm{A}_{101}\right)$ is the most stable and, as a consequence, the majority surface for nanocrystals, where it occupies more than $94 \%$ of the surface area. ${ }^{9}$ 
Photocatalytic processes take place on $\mathrm{TiO}_{2}$ surfaces, most of such processes in solution at the solid/liquid interface. In particular, the interaction with water is crucial for numerous $\mathrm{TiO}_{2}$ technical applications. ${ }^{10,11}$ Ethanol is also of interest as an alternative fuel to produce hydrogen ${ }^{12}$ or as a feedstock for a possible green chemistry. ${ }^{13}$ Further alcohols serve as model molecules to rationalize the photocatalytic mechanism on the $\mathrm{TiO}_{2}$ surfaces.

In order to better understand the photocatalytic events on anatase surfaces it is of utmost importance to comprehend at an atomistic level the interactions of solvent molecules with ideal or defective surface sites. ${ }^{14-18}$ Defects play a key role for photocatalytic processes, ${ }^{19,20}$ whereas both shallow and deep localized trap states can be introduced by crystal defects into the wide band-gap $(3.2 \mathrm{eV})$ of the anatase polymorph. ${ }^{21}$ Resolving the atomic structure of the $\mathrm{A}_{101}$ /solvent interface is experimentally challenging. ${ }^{11,16,22}$ Ab initio simulations are particularly valuable in this context, allowing for the accurate modeling of these systems as a function of varying external conditions. ${ }^{23}$ In fact, Density Functional Theory (DFT) has become the main workhorse for atomistic simulations, providing a high predictive power for structural properties in materials science at an affordable computational cost.

However, previous studies of water and ethanol adsorption on the anatase $\mathrm{A}_{101}$ surface have been performed in gas-phase conditions, mostly combined with Scanning Tunneling Microscopy (STM) measurements. ${ }^{10,24-27}$ It is important to go beyond gas-phase-like firstprinciple simulations and to include the effects of a wet environment in order to rationalize the interactions and processes at the solid/liquid interface. It is also important to fully explore the configurational space of the molecule/surface system in the actual wet environment. A straightforward approach is to include explicitly all solvent molecules, and treat them at the same level of quantum theory as the solvated system. This explicit inclusion should in principle be the most accurate to account for solvent effects, albeit fully explicit ab initio approaches result in well-known limitations in describing a liquid environment like water, in particular regarding its structural and dielectric properties. ${ }^{28-31}$ Another limiting factor for the explicit treatment is the inefficient sampling of the configurational space, where the 
global search gives back many different configurations of the solvent molecules for identical solvated structures. These detrimental drawbacks hinder the application of powerful structure prediction algorithms to systems immersed in wet environments. The alternative is an implicit description of the solvent effects, while still treating the other parts of the system explicitly at an atomic level.

We include the solvent effects into the DFT calculations by means of the soft-sphere continuum solvation model. ${ }^{32-34}$ The implicit way for structure predictions is appealing since the number of degrees of freedom does not vary with respect to the corresponding gas-phase simulation. To explore the molecule/surface configurational space and the various adsorption mechanisms at the $\mathrm{A}_{101}$ /solvent interface, we couple the Minima Hopping $(\mathrm{MH})$ structure prediction method ${ }^{35}$ with the soft-sphere model.

Our proposed paradigm sheds light on the interaction of both water and ethanol at the $\mathrm{A}_{101}$ solid/liquid interface. The implicit approach correctly reproduces surface atomic displacements extracted from previous experimental Surface X-Ray Diffraction (SXRD) measurements and DFT calculations with explicit solvent molecules. ${ }^{16}$ The stability of oxygen vacancy defects lying at surface sites is strongly affected by the wet environment and the polar character of the solvent. Ab initio Molecular Dynamics (MD) simulations with explicit water molecules support the vacancy energetics. We found that while ethanol molecules are able to passivate surface oxygen vacancies at the solid/liquid $\mathrm{TiO}_{2}$ (101) interface, water molecules only weakly interact with this surface.

We validate our computational approach by Fourier Transform Infrared (FT-IR) and Photoluminescence (PL) spectra of anatase $\mathrm{TiO}_{2}$ nanoparticles exposing predominantly (101) surfaces on water and ethanol solvents. Infrared spectra give direct access to chemical bonds and reactions taking place at surface sites. Photoluminescence spectroscopy is a sensitive tool for probing surface reactions and molecular adsorption in photocatalytic materials, since radiative recombination takes place in a tiny region (few tenths of nanometers wide) below the photocatalytic surface. Furthermore, the investigation of the PL mechanism can deliver 
fundamental information, for example, on the photocatalytic properties of surfaces. The high surface area to volume ratio of nanometer-sized particles allows to highlight the contribution of surface chemistry, its interface with a solvent, and the role of surface defects. As a consequence, $\mathrm{TiO}_{2}$ nanoparticles represent a valuable system for exploring properties and interactions at solid/liquid interfaces. The influence of different contacting fluids with different dielectric constants provide insight in the local atomistic interactions at solid/liquid interfaces as a function of the solvent polarity. Our reported IR and PL measurements on anatase $\mathrm{TiO}_{2}$ nanoparticles confirm the structural predictions for the solvated (1 01 1) anatase surface.

\section{Methods}

The Kohn-Sham density functional theory has been employed within the BigDFT package, ${ }^{36,37}$ using wavelets as basis functions. Wavelets are a fully systematic and localized basis set. As such they combine the advantages of Gaussian and plane wave basis sets. Thanks to their adaptivity, wavelets offer a much better speed and lower memory requirements than plane waves for free boundary conditions. BigDFT uses an adaptive mesh of grid points carrying wavelets and/or scaling functions to create three regions of accuracy: a high resolution is used in regions where the chemical bonding takes place. In the tail regions of the wave functions, where they smoothly decay to zero, a lower resolution is used. Finally, in regions where the wave functions are smaller than a very small value no grid points are used. Compared to plane waves this corresponds to plane waves with a four times higher cutoff energy. Thanks to the integrated Poisson solver based on a Green function formalism, periodic systems, surfaces and isolated systems can be simulated with the proper boundary conditions $(\mathrm{BC})$. It is worth noting that solutes plunged in a solvent or at solid/liquid interfaces always request nonperiodic BCs. Surface boundary conditions impose periodic boundary conditions at the surface plane and free boundary conditions in the direction or- 
thogonal to the surface. Surface BC delivers the exact solution of the DFT problem, free of spurious interactions between the periodic images in the direction orthogonal to the surface which are present for full periodic BC like in plane wave approaches. We used surface BC for all calculations on our slab models.

Complex wet environments have been included by means of the soft-sphere implicit solvation model introduced in Refs. [32-34]. Soft norm-conserving pseudo-potentials including non-linear core corrections ${ }^{38,39}$ along with the Perdew-Burke-Ernzerhof functional were used to describe the core electrons and exchange-correlation as implemented in the Libxc ${ }^{40}$ library. To make energetic comparisons, we performed all runs with equivalent parameter settings and convergence criteria. All structures were relaxed using the Hellman-Feynman scheme until forces were less than $5.0 \mathrm{meV} / \AA$.

The minima hopping method ${ }^{35}$ was employed to sample low energy molecule-surface configurations by systematically exploring the potential energy surface both in vacuum and with the inclusion of implicit solvents. Within $\mathrm{MH}$, consecutive short molecular dynamics simulations are performed to escape from the local minima, followed by local geometry optimizations to efficiently sample the energy landscape. All structural searches were performed directly at the DFT level by coupling the MH with the BigDFT package. MH was successfully applied in similar applications to predict reconstructions of fluorite surfaces in vacuum and aqueous environments, ${ }^{41}$ as well as the molecular doping of the silicon (111) surface. ${ }^{42}$

The lattice parameters of the conventional anatase cell were optimized using a $4 \times 4 \times 2$ $k$-point Monkhorst-Pack grid until the pressure converged within 0.05 kbar, obtaining a cell with lattice constants $a=3.79 \AA$ and $b=9.71 \AA$. The experimental lattice constants are $a=3.79 \AA$ and $b=9.625 \AA .{ }^{43}$ The anatase $\mathrm{TiO}_{2}$ (101) surface was modeled with six trilayers of a $4 \times 1$ supercell ( 288 atoms in total), starting from the optimized bulk lattice parameters with periodic dimensions of $15.18 \AA$ and $10.43 \AA$. Atoms at the bottom (nonhydrated) trilayer of the slab were fixed at their bulk coordinates to emulate bulk behaviour. Fig. 1 shows the first two trilayers of the $A_{101}$ surface. The $A_{101}$ surface shows a saw- 
tooth-like geometry with five-fold $\left(\mathrm{Ti}_{5 \mathrm{c}}\right)$ and six-fold $\left(\mathrm{Ti}_{6 \mathrm{c}}\right)$ coordinated titanium atoms and two-fold $\left(\mathrm{O}_{2 \mathrm{c}}\right)$ and three-fold $\left(\mathrm{O}_{3 \mathrm{c}}\right)$ coordinated oxygen atoms arranged in a row along the [010] direction. ${ }^{44,45}$ Various slab models with different numbers of O-Ti-O trilayers have been tested in the literature, and computational results (binding energy for adsorbed molecules, defect formation energy) strongly depend on this choice. ${ }^{25}$ A six trilayer slab and a $4 \times 1$ supercell with a fixed bottom layer has provided a fully reliable balance between computational efficiency and accuracy. ${ }^{20,25,46}$ The $4 \times 1$ periodicity prevents spurious interactions with periodic images of the defects or the adsorbed organic molecule in the periodic directions $x$ and $y$ ( $z$ is considered orthogonal to the surface plane, i.e. along the [101] crystal direction). Surface boundary conditions, where periodic replicas for the atomistic system are imposed only on the $x$ and $y$ surface directions, have been set for all surface calculations. The wavelet basis functions were distributed on an adaptive uniform mesh with a resolution of $h_{\text {grid }}:=h_{x}=h_{y}=h_{z}=0.40$ Bohr for all calculations. Due to the large sizes of the system, only the $\Gamma$ point has been used for the $k$-space integration in all geometry optimizations. The energetics have been extracted for the final relaxed structures using a $3 \times 4 \times 1 k$-point Monkhorst-Pack mesh.

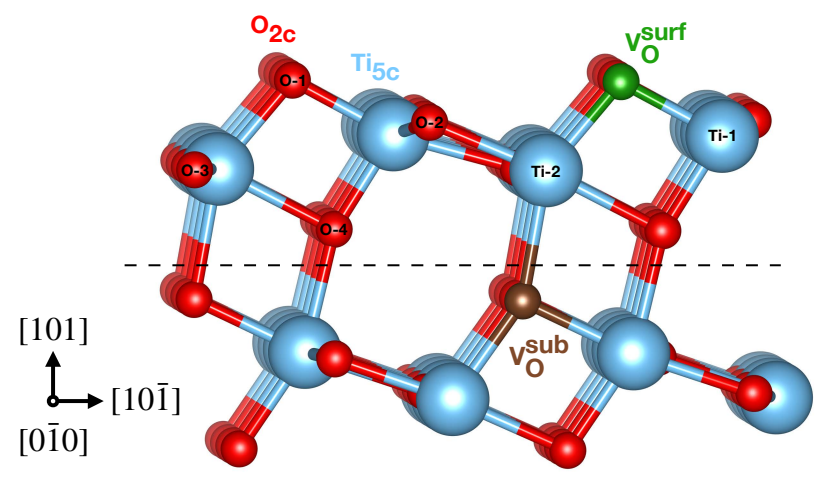

Figure 1: Ball and stick model of the $4 \times 1$ supercell for the anatase $\mathrm{TiO}_{2}$ (101) surface. The numerical labeling supports the identification of atoms for the surface atomic displacements in Table 1. Oxygen: red; Titanium: cyan; Surface oxygen vacancy $\mathrm{V}_{\mathrm{O}}^{\text {surf: }}$ green; Subsurface oxygen vacancy $\mathrm{V}_{\mathrm{O}}^{\mathrm{sub}}$ : brown.

In the soft-sphere continuum solvation model ${ }^{33}$ the interface between the quantum- 
mechanical solute and surrounding environment is described by a fully continuous and differentiable permittivity $\epsilon(\mathbf{r})$, which is a function of the atomic coordinates. $\epsilon(\mathbf{r})$ acquires a value of 1 where the solute system is placed (and where a quantum mechanical approach is applied) and that of the bulk dielectric constant of the surrounding solvent outside. The soft-sphere model improves previous solvation approaches. ${ }^{47}$ The model features are: accurate forces and a numerical cost comparable to standard vacuum calculations; feasible extensive PES explorations; a small number of model parameters; exact treatment of molecular or slab-like geometries; ability to treat neutral and charged molecules simultaneously in order to tackle complex interfaces (e.g. a double layer). The interface between the quantum-mechanical solute and the surrounding environment is described by a fully continuous permittivity built up with atomic-centered "soft" spheres. This approach combines many of the advantages of the self-consistent continuum solvation model ${ }^{48}$ in handling solutes and surfaces in contact with complex dielectric environments or electrolytes in electronic-structure calculations. In addition, it is able to describe accurately both neutral and charged systems. The continuous function, describing the variation of the permittivity, allows to compute analytically the non-electrostatic contributions to the solvation free energy that are described in terms of the quantum surface. The capability to treat arbitrary molecular or slab-like geometries as well as charged molecules is key to tackle electrolytes within mixed explicit/implicit frameworks. Within the soft-sphere model two parameters are sufficient to give a mean absolute error of only $1.12 \mathrm{kcal} / \mathrm{mol}$ with respect to the experimental aqueous solvation energies for a set of 274 neutral solutes.

The implicit solvent has been preferred to the explicit treatment in order to keep the size of the whole system affordable at a DFT level, without neglecting the solid-liquid interaction. When solvent effects are considered, one surface is considered to be in contact with the implicit solvent. When adsorption is investigated, this is the surface where the solvent molecules are eventually attached. For the opposite (bottom) surface, vacuum conditions are always used by setting a large radius of the soft-sphere model for bottom atoms. This 
guarantees that bottom atoms are never in contact with the implicit solvent. Water and ethanol have, respectively, a dielectric constant of 78.36 and 24.85 at $T=20^{\circ} \mathrm{C}$. ${ }^{49}$ The softsphere model parameters are reported in Ref. [ 33].

\subsection{Theoretical assessment of the solid/liquid interface}

In order to assess the implicit approach for the solid/liquid interface, we extracted surface atomic displacements along $y=[10 \overline{1}]$ and $z=[101]$ directions for the anatase $(101)$ surface in contact with water and ethanol. Starting from a cleaved bulk terminated structure of $\mathrm{A}_{101}$, we optimized the surface in vacuum and in contact with implicit solvents. Nadeem $e t$ al. ${ }^{16}$ investigated $\mathrm{A}_{101}$ atomic surface displacements by means of experimental surface X-ray diffraction and DFT calculations, both in ultrahigh vacuum (UHV) and after a formation of a water interface. In their study, DFT data have been computed with explicit water molecules. As a consequence, such displacement dataset represents a perfect benchmark for our implicit solvation model.

Table 1 reports all surface atomic displacements for oxygen and titanium atoms of the outermost trilayer. The atomic numbering is the one from Fig. 1. The numbers increase for atoms further away from the solid/liquid interface, i.e. towards the $\mathrm{TiO}_{2}$ bulk.

A general agreement of our calculation in vacuum with the experimental SXRD and DFT data of Ref. [ 16] can be noticed, both for the $\Delta y$ and $\Delta z$ directions. In vacuum a relaxation of surface atoms away from the bulk takes place. Surface atomic displacements at vacuum interface also agree with previous DFT calculations. ${ }^{9,46}$

Atomic displacements at the $\mathrm{A}_{101}$ /water interface described by the implicit solvent are consistent with SXRD and DFT data reported in Ref. [16]. Displacements at aqueous interface are smaller, and then closer to zero, with respect to the interface with vacuum. The interface with the implicit solvent yields a $\Delta z$ displacement for the oxygen atoms similar to both experimental SXRD and DFT calculations with explicit water molecules. For the more external surface sites (O-1, O-2, O-3, and Ti-1) $\Delta y$ displacements are similar in magnitude 
Table 1: Surface atomic displacements $[\AA]$ away from the bulk terminated structure of $A_{101}$, where $y=[10 \overline{1}]$ and $z=[101]$. Each single entry represents, respectively: experimental SXRD data (Ref. [ 16]); DFT data with explicit water molecules (Ref. [ 16]); DFT data from the current work with the implicit solvent. See Fig. 1 for atomic numbering.

\begin{tabular}{|c|c|c|c|c|c|c|}
\hline \multirow{3}{*}{ atom } & \multicolumn{2}{|c|}{$\mathrm{A}_{101} /$ vacuum } & \multicolumn{2}{|c|}{$\mathrm{A}_{101} /$ water } & \multicolumn{2}{|c|}{$\mathrm{A}_{101} /$ ethanol } \\
\hline & $\Delta y$ & $\Delta z$ & $\Delta y$ & $\Delta z$ & $\Delta y$ & $\Delta z$ \\
\hline & {$\left[\mathrm{SXRD}^{a} ; \mathrm{DFT}^{b} ; \mathrm{DFT}^{c}\right]$} & {$\left[\mathrm{SXRD}^{a} ; \mathrm{DFT}^{b} ; \mathrm{DFT}^{c}\right]$} & {$\left[\mathrm{SXRD}^{a} ; \mathrm{DFT}^{d} ; \mathrm{DFT}^{e}\right]$} & {$\left[\mathrm{SXRD}^{a} ; \mathrm{DFT}^{d} ; \mathrm{DFT}^{e}\right]$} & {$\left[\mathrm{DFT}^{e}\right]$} & {$\left[\mathrm{DFT}^{e}\right]$} \\
\hline O-1 & $0.14 ; 0.23 ; 0.24$ & $0.10 ; 0.02 ; 0.046$ & $-0.03 ;-0.05 ; 0.08$ & $0.03 ; 0.05 ; 0.03$ & 0.18 & 0.04 \\
\hline O-2 & $0.13 ; 0.14 ; 0.14$ & $0.14 ; 0.25 ; 0.28$ & $-0.03 ;-0.04 ; 0.03$ & $0.09 ; 0.17 ; 0.17$ & 0.09 & 0.24 \\
\hline O-3 & $0.16 ; 0.11 ; 0.12$ & $0.05 ; 0.06 ; 0.09$ & $-0.04 ;-0.09 ; 0.03$ & $0.04 ; 0.01 ; 0.01$ & 0.08 & 0.06 \\
\hline O-4 & $0.01 ; 0.13 ; 0.13$ & $0.01 ;-0.02 ; 0.00$ & $0.04 ;-0.05 ; 0.08$ & $0.01 ; 0.08 ; 0.03$ & 0.11 & 0.01 \\
\hline Ti-1 & $0.02 ;-0.01 ;-0.005$ & $-0.01 ;-0.12 ;-0.09$ & $-0.02 ;-0.06 ;-0.09$ & $0.11 ; 0.13 ;-0.01$ & -0.04 & -0.06 \\
\hline Ti-2 & $0.11 ; 0.12 ; 0.13$ & $0.16 ; 0.21 ; 0.23$ & $0.02 ;-0.07 ; 0.03$ & $0.06 ; 0.03 ; 0.14$ & 0.09 & 0.20 \\
\hline
\end{tabular}

${ }^{a}$ Experimental SXRD data from Ref. [ 16]); ${ }^{b}$ DFT data from Ref. [ 16]); ${ }^{c}$ DFT data from current work); ${ }^{d}$ DFT data with explicit water molecules from Ref. [ 16]); ${ }^{e}$ DFT data with implicit solvent from current work).

but with an opposite sign. Discrepancies can be ascribed to the intrinsic differences between the explicit and implicit treatment of the solvent. Indeed, with the implicit approach atoms and/or molecules composing the liquid will be, on average, everywhere around the solvated system and represent an average of all possible solvent configurations. On the other side, a single DFT run with explicit water molecules freezes in a particular liquid configuration, and does not account for the necessary statistical sampling of various structural arrangements.

Our implicit treatment does not fully recover the $\Delta z$ relaxation of the five-fold coordinate titanium atom Ti-1 at the $\mathrm{A}_{101}$ surface, which is present in the experimental SXRD data or DFT calculations with explicit water. Such movement is attributed to the Ti-1 bonds to the oxygen atom of $\mathrm{H}_{2} \mathrm{O}$ or $\mathrm{OH}$. Data of Nadeem et al ${ }^{16}$ show that $75 \%$ of water molecules are dissociated for a thick layer of water at room temperature. If we add one explicit water molecule to the Ti- 1 surface site we recover a value of $0.074 \AA$ and $0.003 \AA$ for molecular adsorption in vacuum and implicit solvent, respectively. Once a hydroxyl radical HO binds 
to Ti-1, the latter moves by $0.42 \AA$ and $0.29 \AA$ in vacuum and implicit solvent, respectively. Considering $75 \%$ of dissociated water molecules, we can extrapolate an average value of 0.21 $\AA$ Ti-1 z-displacement. Surface atomic displacements for the $\mathrm{A}_{101}$ /ethanol interface lie in between vacuum and water data. This trend properly reflects the relative dielectric constants of the different medium. The higher the solvent permittivity is, the larger is the variation with respect to the vacuum conditions.

$\mathrm{Ti}_{5 \mathrm{c}}$ and $\mathrm{O}_{2 \mathrm{c}}$ are the active sites of the $\mathrm{A}_{101}$ surface, where $\mathrm{Ti}_{5 \mathrm{c}}$ can bind to $\mathrm{H}_{2} \mathrm{O}$ or $\mathrm{OH}$ whilst $\mathrm{O}_{2 \mathrm{c}}$ to a proton on a $\mathrm{A}_{101}$ /water interface. Our calculations show a $\mathrm{Ti}_{5 \mathrm{c}}-\mathrm{O}_{2 \mathrm{c}}$ bond length of $1.84 \AA$ in vacuum and $1.86 \AA$ in contact with implicit water. Ref. [ 50] reports a $\mathrm{Ti}_{5 \mathrm{c}}-\mathrm{O}_{2 \mathrm{c}}$ bond length of $1.86 \AA$ for a clean surface and $1.88 \AA$ in the presence of explicit $\mathrm{H}_{2} \mathrm{O}$ or $\mathrm{OH}$ at the $\mathrm{Ti}_{5 \mathrm{c}}$ site. However, such bond length increases up to $\sim 2.0 \AA$ once a hydrogen atom is bound to the $\mathrm{O}_{2 \mathrm{c}}$ site. ${ }^{16}$ In the latter case, we recover a value of 1.99 and $2.02 \AA$ at vacuum and aqueous interface, respectively.

In colclusion, the present results suggest that the implicit approach for the $\mathrm{A}_{101} /$ water interface properly matches all trends extracted from experimental SXRD measurements and DFT calculations with explicit water molecules.

\section{$3 \quad$ Results and Discussion}

In this paragraph we report simulation results for the water and ethanol adsorption on the perfect and defective $A_{101}$ surface. After the energetic analysis of surface and subsurface oxygen vacancy defects lying at the solid/liquid interface, we explore the adsoption of a single water and ethanol molecule on the wet $\mathrm{A}_{101}$ surface.

\subsection{Oxygen vacancy formation energies}

Vacancy defects play a fundamental role for the $\mathrm{TiO}_{2}$ surface reactivity. Anatase (101) surface shows a reduced room temperature reactivity for the adsorption and dissociation of 
water molecules with respect to the rutile $(110)^{51}$. This is thought to be related to the minor concentration of surface oxygen vacancies at $A_{101}$ termination with respect to the rutile (110) surface. We consider two surface defects: an oxygen vacancy at the outermost $\mathrm{O}_{2 \mathrm{c}}$ surface site belonging to the first trilayer (named surface vacancy or $\mathrm{V}_{\mathrm{O}}^{\text {surf }}$ ); an oxygen vacancy at the bulk lattice site belonging to the second trilayer (named subsurface vacancy or $\left.\mathrm{V}_{\mathrm{O}}^{\mathrm{sub}}\right)$. Both oxygen vacancies are depicted in Fig. 1.

We calculated formation energies of an oxygen vacancy defect as

$$
E_{\text {form }}\left(\mathrm{V}_{\mathrm{O}}\right)=E_{\text {tot }}(\text { def })-E_{\text {tot }}(\text { no def })+\frac{1}{2} \mu\left(\mathrm{O}_{2}\right)
$$

where $E_{\text {tot }}($ def $)$ and $E_{\text {tot }}$ (no def) represent the total energy of defective and stoichiometric anatase $A_{101}$ surface, respectively. The chemical potential of $\mu\left(\mathrm{O}_{2}\right)$ is taken to be the total energy of an isolated $\mathrm{O}_{2}$ molecule. All energetics refer to the final relaxed structures in the proper environment, i.e. vacuum or in the presence of water or ethanol.

For the $\mathrm{A}_{101} /$ vacuum interface we extracted a surface energy of $0.50 \mathrm{~J} / \mathrm{m}^{2}$, in agreement with the value of $0.44 \mathrm{~J} / \mathrm{m}^{2}$ reported by Lazzeri et al. ${ }^{52}$ Table 2 reports formation energies for $\mathrm{V}_{\mathrm{O}}^{\text {surf }}$ and $\mathrm{V}_{\mathrm{O}}^{\text {sub }}$. When $\mathrm{A}_{101}$ forms an interface with vacuum, $E_{\text {form }}\left(\mathrm{V}_{\mathrm{O}}^{\text {sub }}\right)<E_{\text {form }}\left(\mathrm{V}_{\mathrm{O}}^{\text {surf }}\right)$ by $0.40 \mathrm{eV}$. Formation energies are $5.06 \mathrm{eV}$ for $\mathrm{V}_{\mathrm{O}}^{\text {surf }}$, and $4.66 \mathrm{eV}$ for $\mathrm{V}_{\mathrm{O}}^{\text {sub }}$. As a consequence, a subsurface vacancy is energetically favorable with respect to surface vacancies at $\mathrm{O}_{2 c}$ sites. This result agrees with previous DFT studies using the Perdew-Burke-Ernzerhof functional where the formation energy of a subsurface vacancy on anatase $\mathrm{TiO}_{2}\left(\begin{array}{lll}1 & 0 & 1\end{array}\right)$ surface was found to be $0.40 \mathrm{eV}$ lower than at $\mathrm{O}_{2 \mathrm{c}}$ surface sites. ${ }^{19,20,53} \mathrm{~A}$ similar result has been reproduced with a hybrid screened exchange functional, obtaining a difference in stability of $0.30 \mathrm{eV} .{ }^{53}$

Table 2: Vacancy formation energies $E_{\text {form }}\left(\mathrm{V}_{\mathrm{O}}\right)[\mathrm{eV}]$ at the $\mathrm{A}_{101}$ surface.

\begin{tabular}{cccc}
\hline \hline & $\mathrm{A}_{101} /$ vacuum & $\mathrm{A}_{101} /$ water & $\mathrm{A}_{101} /$ ethanol \\
\hline $\mathrm{V}_{\mathrm{O}}^{\text {surf }}$ & 5.06 & 3.97 & 4.59 \\
$\mathrm{~V}_{\mathrm{O}}^{\text {sub }}$ & 4.66 & 4.59 & 4.61 \\
\hline \hline
\end{tabular}


For the $\mathrm{A}_{101} /$ water interface the situation is reversed: $E_{\text {form }}\left(\mathrm{V}_{\mathrm{O}}^{\text {surf }}\right)<E_{\text {form }}\left(\mathrm{V}_{\mathrm{O}}^{\text {sub }}\right)$ by 0.62 $\mathrm{eV}$. Formation energies are $3.97 \mathrm{eV}$ for $\mathrm{V}_{\mathrm{O}}^{\text {surf }}$, and $4.59 \mathrm{eV}$ for $\mathrm{V}_{\mathrm{O}}^{\text {sub }}$. Vacancy defects at the $\mathrm{A}_{101} /$ ethanol interface are energetically equivalent, i.e. $E_{\mathrm{form}}\left(\mathrm{V}_{\mathrm{O}}^{\text {surf }}\right) \sim E_{\text {form }}\left(\mathrm{V}_{\mathrm{O}}^{\text {sub }}\right)$, with $\mathrm{V}_{\mathrm{O}}^{\text {surf }}$ lower than $\mathrm{V}_{\mathrm{O}}^{\text {sub }}$ by $26 \mathrm{meV}$. Formation energies are $4.59 \mathrm{eV}$ for $\mathrm{V}_{\mathrm{O}}^{\text {surf }}$, and $4.61 \mathrm{eV}$ for $\mathrm{V}_{\mathrm{O}}^{\text {sub }}$. The behavior of oxygen vacancies at the $\mathrm{A}_{101} /$ ethanol interface lies in between vacuum and water effects. When moving from vacuum to ethanol or water, the trends of the energetics for surface and subsurface oxygen vacancies are consistent with the increasing dielectric constant of such medium.

To benchmark the relative stability of surface and subsurface oxygen vacancies in the presence of a water environment obtained with the implicit solvation approach, we performed a molecular dynamics simulation of the Anatase $\mathrm{TiO}_{2}\left(\begin{array}{lll}1 & 0 & 1\end{array}\right)$ interface with explicit water molecules. We used the same slab model reported in Section 2, i.e. a six trilayers slab of a $4 \times 1$ supercell $\left(288\right.$ atoms in total). The slab contains a subsurface oxygen vacancy $\mathrm{V}_{\mathrm{O}}^{\text {sub }}$ at the bulk lattice site belonging to the second trilayer (see Fig. 1). We impose periodic boundary conditions. Slabs are separated by a space of $10 \AA$ leading to an orthorhombic supercell of $15.18 \times 32.09 \times 10.42 \AA^{3}$. To model the $\mathrm{A}_{101} /$ water interface, the empty space between the two $A_{101}$ slab surfaces (lower and upper surface) is fully filled with 53 water molecules, leading to two water $/ \mathrm{TiO}_{2}$ interfaces in each unit cell (446 atoms for the whole $\mathrm{A}_{101} /$ water system). Fig. 2a shows a snapshot of the molecular dynamics supercell. The number of water molecules has been chosen to adjust the effective density of the water layer to the ambient density of water. Soft norm-conserving pseudo-potentials including non-linear core corrections along with the Perdew-Burke-Ernzerhof functional were used to describe the core electrons and exchange-correlation. The wavelet basis functions were distributed on an adaptive uniform mesh with a resolution of $h_{\text {grid }}:=h_{x}=h_{y}=h_{z}=0.40$ Bohr. Due to the large sizes of the system, only the $\Gamma$ point has been used for the $k$-space integration during the MD trajectory. The molecular dynamics simulations use the Born-Oppenheimer method and have been carried out using the BigDFT package. The time step for the MD 
simulation was 0.5 fs. NVT conditions were imposed by a Nose-Hoover thermostat with a target temperature of $330 \mathrm{~K}$. The temperature of $330 \mathrm{~K}$ is chosen to avoid the glassy behavior of PBE liquid water on the 20 ps time scale observed for trajectories at lower temperatures. ${ }^{30}$ In MD runs, 1-2 ps of equilibration period is followed by 5 ps of production period.

We started two different molecular dynamics runs: in the first run one of the solvent molecules is placed in the proximity of a $\mathrm{Ti}_{5 c}$ site, which is second nearest-neighbor of the $\mathrm{V}_{\mathrm{O}}^{\text {sub }}$ (Ti-1 atom of Fig. 1) in order to be molecularly adsorbed on such atom; in the second run one of the solvent molecules is dissociated and its $-\mathrm{OH}$ group binds to the $\mathrm{Ti}_{5 \mathrm{c}}$ site, which is second nearest-neighbor of the $\mathrm{V}_{\mathrm{O}}^{\mathrm{sub}}$.

On both exposed surfaces (one perfect and one defective), water forms a layered structure of intact molecules that exhibit orientational order in the first two layers. Water in the first solvation layer coordinates to surface $\mathrm{Ti}_{5 c}$ sites, whilst in the second solvation layer it forms short $\mathrm{H}$-bonds with surface undercoordinated $\mathrm{O}_{2 c}$. Short $\mathrm{H}$-bonds are rarely broken during our MD runs. Such sluggish H-bond networks result in an ice-like dynamics for the first two solvation layers. Beyond the second solvation layer water molecules do not interact directly with the oxide surface and behave like bulk water. Results are in agreement with the study of Calegari Andrade and co-workers ${ }^{18}$ for the hydrated stoichiometric $A_{101}$ surface.

During the first MD run, we do not observe any vacancy movement towards the surface site $\mathrm{V}_{\mathrm{O}}^{\text {surf }}$, depicted as green ball in Fig. 1. Instead, during the first picoseconds of the second MD run, the initial subsurface oxygen vacancy (Fig. 2b) moves up towards the first trilayer (Fig. 2c). The latter represents a metastable intermediate site between $\mathrm{V}_{\mathrm{O}}^{\text {sub }}$ and $\mathrm{V}_{\mathrm{O}}^{\text {surf }}$, named $\mathrm{V}_{\mathrm{O}}^{\text {inter }}$. Finally the vacancy occupies a $\mathrm{V}_{\mathrm{O}}^{\text {surf }}$ surface site (Fig. 2d). Fig. 2b-2d reports the full MD dynamics. A movie is also reported in the Supporting Information.

The diffusion dynamics can be explained by the action of the electronegative oxygen atom of the hydroxyl group, which captures electrons of the $\mathrm{Ti}_{5 \mathrm{c}}$ atom (which is second nearestneighbor of $\mathrm{V}_{\mathrm{O}}^{\mathrm{sub}}$ ), weakening the $\mathrm{Ti}_{5 \mathrm{c}}$ bond to the underlying oxygen atom (nearest-neighbor of $\left.\mathrm{V}_{\mathrm{O}}^{\mathrm{sub}}\right)$. In turn, the latter diffuses at the $\mathrm{V}_{\mathrm{O}}^{\text {sub }}$ site, leaving back a surface oxygen vacancy 
$\mathrm{V}_{\mathrm{O}}^{\text {surf }}$. We cannot exclude that a similar vacancy migration takes place for the first MD run but at a longer time scale. A similar dynamics is expected for an MD simulation of the $\mathrm{A}_{101}$ interface with explicit ethanol molecules since structure predictions for ethanol adsorption suggest that subsurface oxygen vacancies are not stable after ethanol dissociation (see the ethanol adsorption configuration of Fig. 4i with the presence of an oxygen vacancy at an intermediate site $\left.\mathrm{V}_{\mathrm{O}}^{\mathrm{inter}}\right)$.

The fact that a subsurface oxygen vacancy moves spontaneously towards a superficial position during an MD kinetics of a few ps shows that the barrier between the two configurations is very low. Considering the Bell-Evans-Polanyi principle ${ }^{54}$ which states that highly exothermic chemical reactions have a low activation energy, molecular dynamics results with explicit water molecules agree with the implicit description of the solid/liquid interface, where an energy difference of $0.62 \mathrm{eV}$ between surface and subsurface oxygen vacancy configurations is found.

The present results obtained with the implicit solvent, i.e. oxygen vacancies prefer to lie at surface sites $\mathrm{V}_{\mathrm{O}}^{\text {surf }}$ than subsurface $\mathrm{V}_{\mathrm{O}}^{\text {sub }}$ ones, agree with the structure predictions of single molecule adsorption at the defective $A_{101}$ surface reported in the next sections. Subsurface vacancies are destabilised after water or ethanol adsorption in the proximity of the defect site and start to migrate at surface sites. Further, low energy molecule/surface configurations both at vacuum and solvent environments are characterized by the presence of surface vacancies $\mathrm{V}_{\mathrm{O}}^{\text {surf }}$.

\subsection{Water and ethanol adsorption at the $A_{101}$ surface}

In order to get more insight into the interaction of the molecules with the anatase $\mathrm{TiO}_{2}$ (10 1) surface, several molecule/surface configurations have been optimized. We considered both the perfect stoichiometric $A_{101}$ surface and the defective termination with the presence of surface/subsurface oxygen vacancies. The $\mathrm{A}_{101}$ active sites for molecule adsorption are $\mathrm{Ti}_{5 \mathrm{c}}$ and $\mathrm{O}_{2 \mathrm{c}} \cdot{ }^{10,22,26,27,55} \mathrm{Ti}_{5 \mathrm{c}}$ can bind to the oxygen atom of $\mathrm{H}_{2} \mathrm{O}$ and the $\mathrm{OH}$ radicals as well 


\section{(a)}

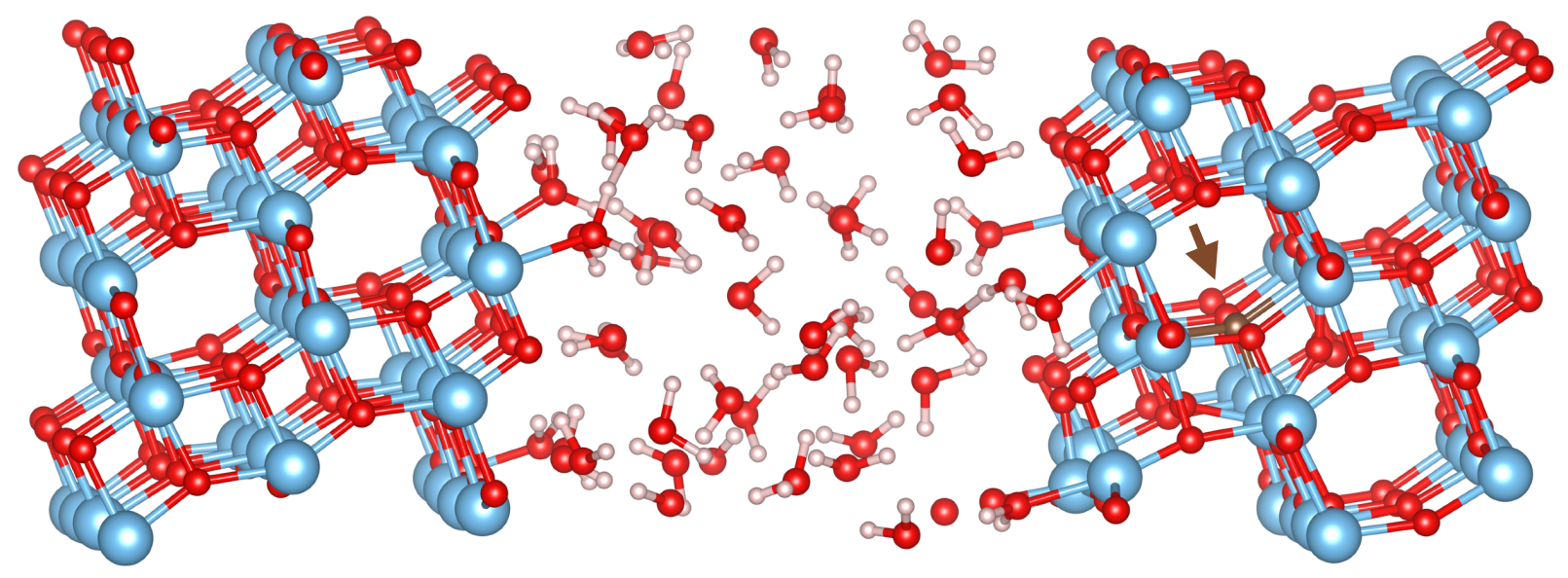

(b)

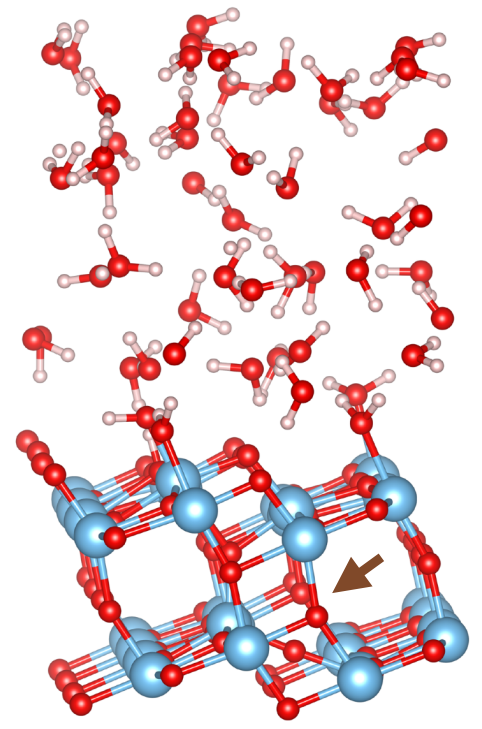

(c)

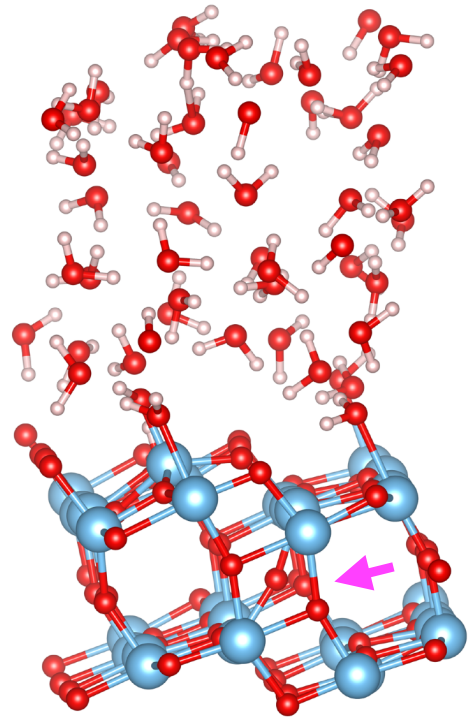

(d)

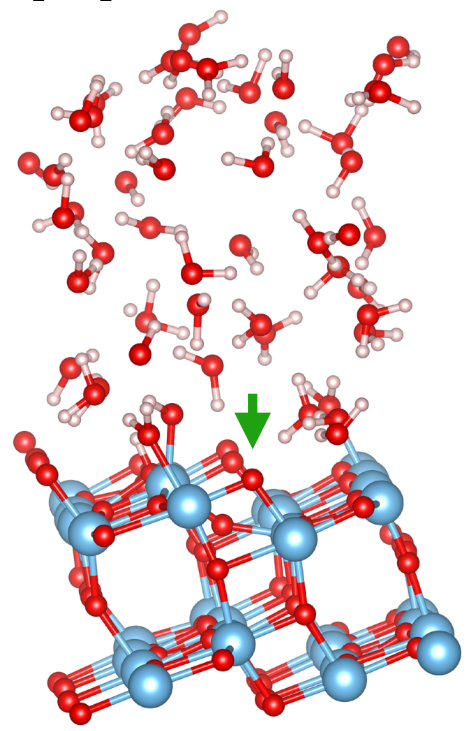

Figure 2: (a) Snapshot of the molecular dynamics supercell used to simulate the anatase-water interface. (b) Initial MD step with the presence of a subsurface oxygen vacancy $\mathrm{V}_{\mathrm{O}}^{\text {sub }}$ (brown arrow). (c) Intermediate MD step where the subsurface oxygen vacancy slightly moves up towards the first trilayer (magenta arrow), occupying the metastable site $V_{O}^{\text {inter }}$. (d) Final MD step where the vacancy occupies a $V_{\mathrm{O}}^{\text {surf }}$ surface site (green arrow). 
as to $\mathrm{CH}_{3} \mathrm{CH}_{2} \mathrm{OH}$ and $\mathrm{CH}_{3} \mathrm{CH}_{2} \mathrm{O}$ groups. $\mathrm{O}_{2 \mathrm{c}}$ binds to the protons generated by dissociative adsorptions.

In order to quantify the interaction between the molecule and the substrate, we computed the molecule binding energy $\Delta E_{\mathrm{b}}$, defined as the difference of the total energy of the system (molecule + surface) and their energies in isolated conditions. The reference systems to compute adsorption energies in vacuum are a slab, an isolated molecule and a slab with an adsorbed molecule (both molecular and dissociative adsorption) in vacuum. The reference systems to compute adsorption energies in a solvent are a slab in contact with the implicit solvent, a molecule immersed in implicit solvent and a slab/liquid interface (liquid described by the implicit model) with an adsorbed molecule. Negative binding energy means that the adsorption is exothermic. All energies have been extracted after relaxation. We consider both molecular and dissociative adsorption. The dissociation of a water molecule produces a proton $\mathrm{H}^{+}$and hydroxyl radical $\mathrm{HO}^{-}$. The most favorable dissociation pathway of ethanol $\mathrm{CH}_{3} \mathrm{CH}_{2} \mathrm{OH}$ on the $\mathrm{A}_{101}$ termination is the $\mathrm{O}-\mathrm{H}$ bond cleavage, followed by ethoxide and proton production. ${ }^{27,56}$

\subsubsection{Perfect surface}

Table 3 reports the binding energy for the water and ethanol molecules at the stoichiometric $\mathrm{A}_{101}$ surface. We consider molecular and dissociative adsorption both at the vacuum and at the solid/liquid interface. We explored the stability of the various configurations where water and ethanol molecules bind to an active $\mathrm{Ti}_{5 \mathrm{c}}$ surface site, and the protons of a dissociated molecule at the $\mathrm{O}_{2 c}$ bridge sites.

Fig. 3 shows adsorption configurations on the non-defected $A_{101}$ surface for water (a and c) and ethanol (b and d). Molecules adsorb with the same configuration both at the $\mathrm{A}_{101} /$ vacuum and at the $\mathrm{A}_{101} /$ solvent interface. At the $\mathrm{A}_{101} /$ vacuum interface, molecular adsorption is favoured with respect to a dissociative process. The binding energy for a water molecule is $-0.70 \mathrm{eV}$ for the molecular adsorption (Fig. 3a), and $-0.32 \mathrm{eV}$ for the dissociative 
case (Fig. 3c). For ethanol, molecular (Fig. 3b) and dissociative (Fig. 3d) processes give a binding energy of $-0.72 \mathrm{eV}$ and $-0.40 \mathrm{eV}$, respectively. Dissociative adsoption for water is characterized by a hydroxyl $\mathrm{OH}$ bound to $\mathrm{Ti}_{5 \mathrm{c}}$ (terminal hydroxyl $\mathrm{OH}_{\mathrm{t}}$ ) and an hydrogen $\mathrm{H}$ bound to the nearest-neighbor $\mathrm{O}_{2 \mathrm{c}}$ bridge (bridging $\mathrm{OH}_{\mathrm{b}}$ ). In paralell, dissociative adsoption for ethanol leads to a ethoxide $\mathrm{OCH}_{2} \mathrm{CH}_{3}$ group bound to $\mathrm{Ti}_{5 \mathrm{c}}$ (terminal ethoxide EtO $\mathrm{Ti}_{\mathrm{Ti}}$ ) and a bridging $\mathrm{OH}_{\mathrm{b}}$.

Table 3: Binding energies $[\mathrm{eV}]$ of water and ethanol molecules on the perfect $\mathrm{A}_{101}$ surface in contact with vacuum and wet environments.

\begin{tabular}{lcccc}
\hline \hline \multirow{2}{*}{$\mathrm{A}_{101}$ surface } & molecule & adsoption mode & \multicolumn{2}{c}{$\Delta E_{\mathrm{b}}$} \\
\cline { 3 - 5 } perfect & & & $\mathrm{A}_{101} /$ vacuum & $\mathrm{A}_{101} /$ solvent \\
& $\mathrm{H}_{2} \mathrm{O}$ & molecular & -0.70 & 0.004 \\
& $\mathrm{H}_{2} \mathrm{O}$ & dissociative & -0.32 & 0.29 \\
& $\mathrm{CH}_{3} \mathrm{CH}_{2} \mathrm{OH}$ & molecular & -0.72 & -0.34 \\
& $\mathrm{CH}_{3} \mathrm{CH}_{2} \mathrm{OH}$ & dissociative & -0.40 & -0.08 \\
\hline \hline
\end{tabular}

The energetics for water adsoption at $\mathrm{A}_{101}$ /vacuum interface agree with previous experimental and computational results. Water was found to adsorb non-dissociatively on the anatase $\left(\begin{array}{lll}1 & 0 & 1\end{array}\right)$ surface, ${ }^{10,24,50,57}$ with the oxygen atom of $\mathrm{H}_{2} \mathrm{O}$ bound to the $\mathrm{Ti}_{5 \mathrm{c}}$ site and its $\mathrm{H}$ atoms forming hydrogen bonds with the two neighboring $\mathrm{O}_{2 c}$ (see Fig. 3a). The reported adsorption energy of $\mathrm{H}_{2} \mathrm{O}$ is $-0.73 \mathrm{eV} .{ }^{10}$ Our data also agree with structures and energetics reported by Vittadini et al. concerning molecular $(-0.74 \mathrm{eV})$ and dissociative $(-0.30 \mathrm{eV})$ adsorption of water at $\mathrm{A}_{101}$ /vacuum interface, ${ }^{50}$ as well as with temperature-programmed desorption spectra, which show a broad water desorption peak centered at $\sim 250 \mathrm{~K}{ }^{24}$

Concerning ethanol adsorption, our results agree with previous DFT calculations 27,56 with close molecular $\left(\mathrm{EtOH}_{\mathrm{Ti}}\right)$ and dissociative adsorption energies at $\mathrm{A}_{101}$ surface in vacuum. Katsiev et al. report that molecular adsorption $(-1.11 \mathrm{eV})$ is energetically favoured 

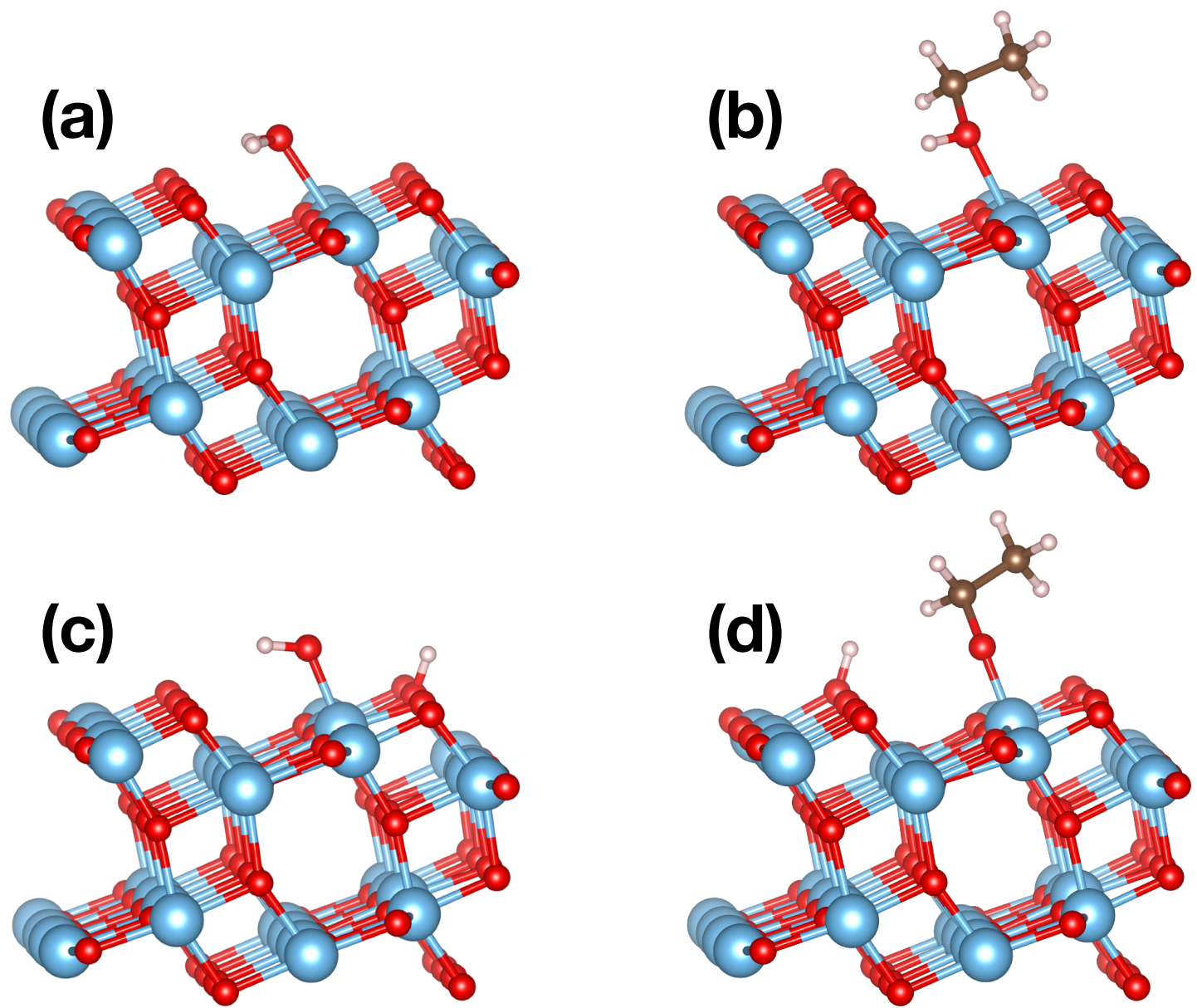

Figure 3: Global minima of molecular ( $\mathrm{a}$ and $\mathrm{b}$ ) and dissociative (c and d) adsorption of water (a and $c$ ) and ethanol ( $b$ and d) on the perfect anatase $\mathrm{Ti}_{2}$ (101) surface. Oxygen: red; Titanium: cyan; Hydrogen: white; Carbon: brown. 
with respect to a dissociative $(-0.93 \mathrm{eV})$ process, with a difference in binding energy of 0.18 eV. ${ }^{27}$ The close energetics between molecular and dissociative adsorption is in agreement with scanning tunneling microscopy measurements of $\mathrm{A}_{101}$ surface exposed to ethanol in ultrahigh-vacuum ${ }^{27}$ conditions. $\mathrm{CH}_{3} \mathrm{CH}_{2} \mathrm{OH}$ as well as $\mathrm{CH}_{3} \mathrm{CH}_{2} \mathrm{O}$ concurrently adsorb at $\mathrm{Ti}_{5 \mathrm{c}}$ surface sites, showing different STM apparent heights of 2.4 and $1.7 \AA$, respectively. Similar results have been found by means of STM measurements and DFT calculations for ethanol adsorption on the rutile $\mathrm{TiO}_{2}$ (110) surface. ${ }^{26}$ The molecular and dissociative adsorption energies on $\mathrm{Ti}_{5 \mathrm{c}}$ sites of rutile (110) are, respectively, $-0.72 \mathrm{eV}$ and $-0.60 \mathrm{eV} .{ }^{26}$ Temperature-programmed desorption analysis revealed a similar prevalent molecular adsorption for methanol at the $A_{101}$ surface in vacuum. ${ }^{24,58}$ The experimental evidence of ethanol diffusion at the $\mathrm{A}_{101}$ surface ${ }^{27}$ or at rutile $\mathrm{TiO}_{2}$ (110) surface ${ }^{26}$ also support the presence of molecular adsorption at UHV conditions.

At the $\mathrm{A}_{101}$ /solvent interface the scenario is unchanged for ethanol, where the solvent slightly weakens the surface/molecule binding both in the molecular and the dissociative adsorption. We recover binding energies of $-0.34 \mathrm{eV}$ and $-0.08 \mathrm{eV}$, respectively. Conversely, the molecular binding energy of $\mathrm{H}_{2} \mathrm{O}$ at the $\mathrm{A}_{101}$ /water is almost zero. Considering eventual computational errors (DFT and solvation model parameters, integration grid), results for water adsorption suggest that if molecular binding is present, at equilibrium it should be weak. As a consequence $\mathrm{H}_{2} \mathrm{O}$ molecules are expected to continuously adsorb/desorb on the $\mathrm{A}_{101} /$ water interface.

\subsubsection{Defective surface}

In this section we focus on a defective nonstoichiometric $A_{101}$ surface with oxygen vacancies on surface $\mathrm{V}_{\mathrm{O}}^{\text {surf }}$ and subsurface $\mathrm{V}_{\mathrm{O}}^{\text {sub }}$ sites. We explore the configurational space of a single solvent molecule adsorbed at various surface sites in the proximity of vacancy defects. We apply the minima hopping method for the structure predictions, both at $\mathrm{A}_{101} /$ vacuum and $\mathrm{A}_{101} /$ solvent interfaces, where in the last case we coupled the $\mathrm{MH}$ with the soft-sphere 
model. In order to reduce the computational load, we modeled the defective $\mathrm{A}_{101}$ slab with a three $\mathrm{O}-\mathrm{Ti}-\mathrm{O}$ trilayer during the $\mathrm{MH}$ runs. Then local minima of the molecule/surface structure have been re-optimized with the same setup of Sec. 2 with a six trilayer slab model. We applied MH starting from various molecule/surface configurations, with the molecule adsorbed at different defective surface sites both in the molecular and dissociative form, considering both $\mathrm{V}_{\mathrm{O}}^{\text {surf }}$ and $\mathrm{V}_{\mathrm{O}}^{\text {sub }}$ at the $\mathrm{A}_{101}$ surface.

Table 4 reports various low energy molecule/surface configurations found by the minima hopping structure prediction algorithm (molecule $=\mathrm{H}_{2} \mathrm{O}, \mathrm{CH}_{3} \mathrm{CH}_{2} \mathrm{OH}$ ) in energetic order starting from the global minimum (GM), i.e. the lowest energy structure found by $\mathrm{MH}$ for a given interface $\left(A_{101} /\right.$ vacuum and $A_{101} /$ solvent). For each particular case (for example $\mathrm{H}_{2} \mathrm{O}$ at $\mathrm{A}_{101}$ /vacuum, molecular and $\mathrm{V}_{\mathrm{O}}^{\text {sub }}$ ) similar structures but higher in energy have been found by the MH method, which differ in the rotation of the molecule or in the adsorption site. In this case we report in Table 4 only the lowest energy configuration. Fig. 4 shows all low energy structures. Colored arrows indicate the position of the surface oxygen vacancy $\mathrm{V}_{\mathrm{O}}^{\text {surf }}$ (green) and the subsurface oxygen vacancy $\mathrm{V}_{\mathrm{O}}^{\text {sub }}$ (brown). The $\mathrm{Ti}^{3+}$ distribution with the lowest energy for the surface and subsurface vacancy agrees with results of MartínezCasado and co-workers. ${ }^{59}$ Structures reported in Fig. 4 correspond to various adsorption configurations of water (a-f, a is the global minimum GM) and ethanol ( $\mathrm{g}-\mathrm{l}$, g is the global minimum). Energy levels $\Delta E[\mathrm{eV}]$ with respect to the global minimum GM are reported (see Table 4 for $\Delta E$ values). Although structures can be metastable (local minima) both in vacuum and in wet conditions, their order can change especially for solvents with high dielectric constant like water. For this reason, in the following, we refer to structures of Fig. 4 to describe also gas-phase configurations.

At the $\mathrm{A}_{101}$ /vacuum interface, $\mathrm{H}_{2} \mathrm{O}$ dissociates with a binding energy of $-1.83 \mathrm{eV}$ (see Fig. 4a). The oxygen atom of the hydroxyl $\mathrm{OH}$ fills the outermost $\mathrm{V}_{\mathrm{O}}^{\text {surf }}$ site, and $\mathrm{H}$ binds to an $\mathrm{O}_{2 \mathrm{c}}$ surface bridge. This configuration forms two bridging hydroxyl groups $\mathrm{OH}_{\mathrm{b}}$. It represents also the $\mathrm{H}_{2} \mathrm{O}$ GM at the $\mathrm{A}_{101}$ /solvent interface. Dissociation at $\mathrm{V}_{\mathrm{O}}^{\text {surf }}$ sites, with 

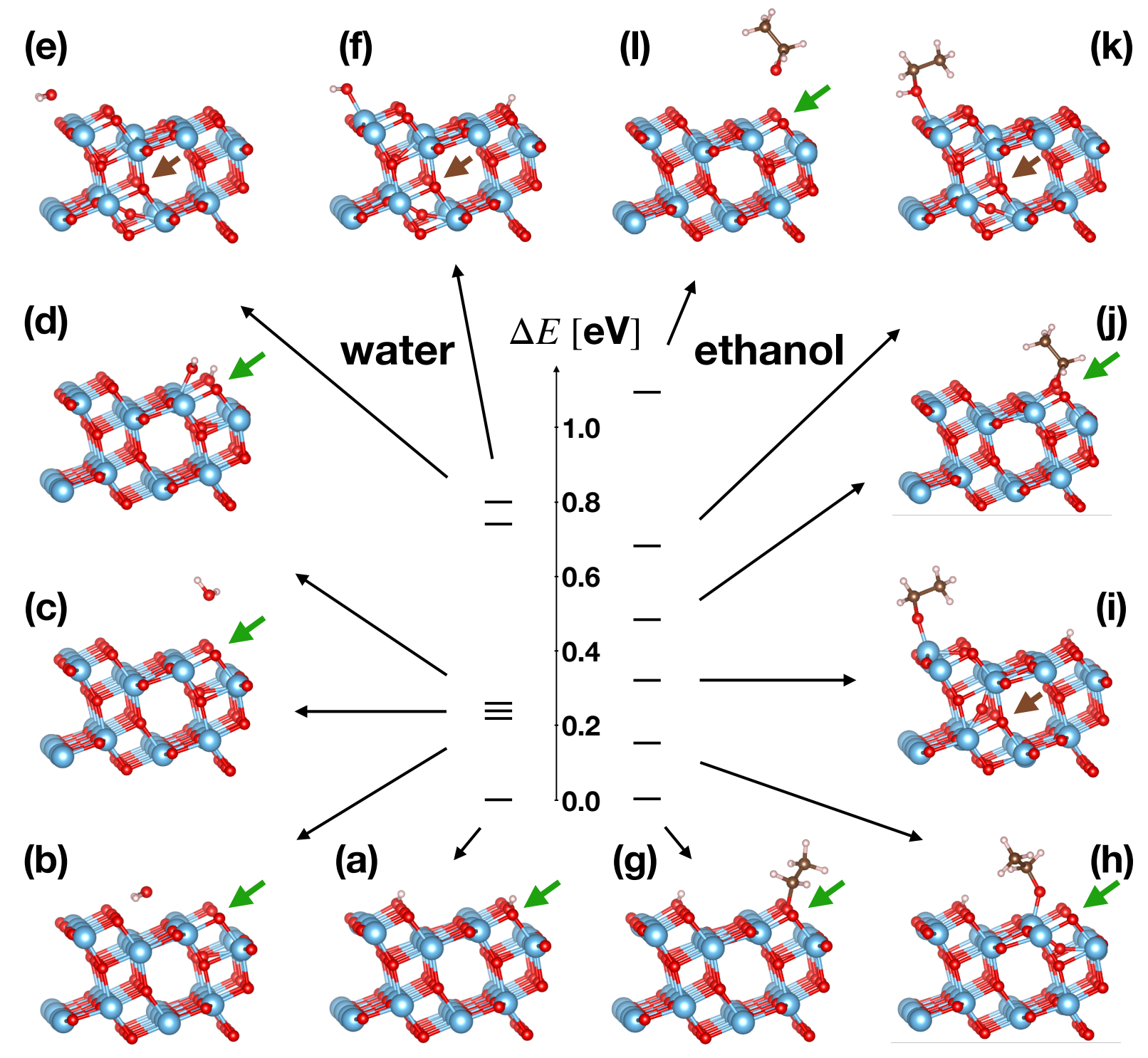

Figure 4: Low energy structures found by the minima hopping structure prediction algorithm for various adsorption configurations of water (a-f, a is the global minimum) and ethanol ( $\mathrm{g}-\mathrm{l}, \mathrm{g}$ is the global minimum) at the $\mathrm{A}_{101}$ defective surface in contact with a wet environment. Energy levels $\Delta E[\mathrm{eV}]$ with respect to the global minimum GM are reported (see Table 4 for $\Delta E$ values). Oxygen: red; Titanium: cyan; Hydrogen: white; Carbon: brown. Colored arrows indicate the position of the surface oxygen vacancy $\mathrm{V}_{\mathrm{O}}^{\text {surf }}$ (green) and the subsurface oxygen vacancy $\mathrm{V}_{\mathrm{O}}^{\text {sub }}$ (brown). 
Table 4: Low energy structures found by the minima hopping structure prediction algorithm for various adsorption configurations of water and ethanol at the $\mathrm{A}_{101}$ defective surface in contact with vacuum and wet environments. Energy for the case where the molecule is away (m.a.) from the surface is included. Each column reports: Energy difference $\Delta E[\mathrm{eV}]$ with respect to the global minimum GM; molecular (mol) or dissociate (dis) state; vacancy defect type; binding energy $\Delta E_{\mathrm{b}}$ $[\mathrm{eV}]$.

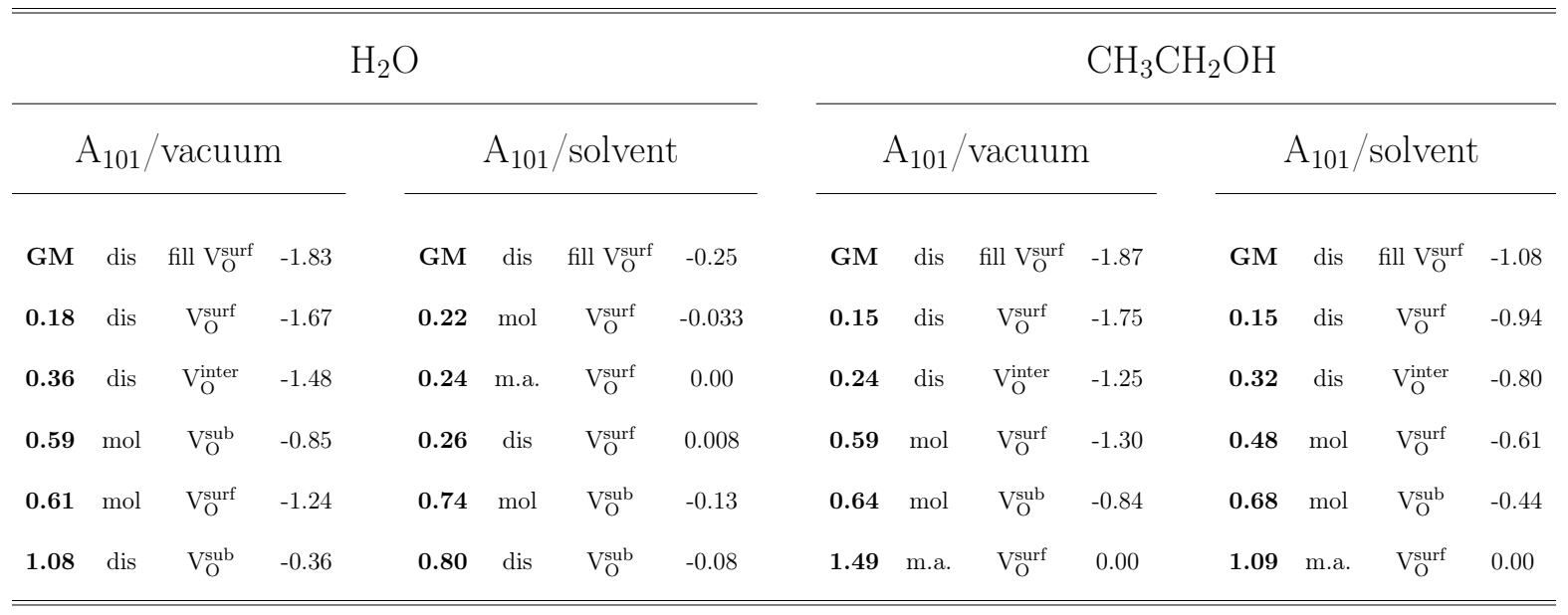

the oxygen atom of the hydroxyl $\mathrm{OH}$ binding only to the four-fold coordinate Ti nearestneighbor of the oxygen vacancy (forming a terminal hydroxyl $\mathrm{OH}_{\mathrm{t}}$ ) and the $\mathrm{H}$ binding to the nearest-neighbor $\mathrm{O}_{2 c}$ (bridging $\mathrm{OH}_{\mathrm{b}}$ ), is $0.18 \mathrm{eV}$ higher in energy with respect to the previous GM (Fig. 4d). This configuration represents a local minimum, i.e. a crossing point before falling to the GM after diffusion of the hydrogen from $\mathrm{OH}_{\mathrm{b}}$.

If $\mathrm{H}_{2} \mathrm{O}$ dissociates at a $\mathrm{Ti}_{5 \mathrm{c}}$ site, which is second nearest-neighbor of a $\mathrm{V}_{\mathrm{O}}^{\text {sub }}$ (Ti-1 atom of Fig. 1), the subsurface oxygen vacancy slightly moves up towards the first trilayer in the metastable configuration named $\mathrm{V}_{\mathrm{O}}^{\text {inter }}$. The same intermediate configuration has been also found during the molecular dynamics trajectory of the $\mathrm{A}_{101}$ interface with explicit water (see the MD snapshot of Fig. 2c). This configuration is $0.36 \mathrm{eV}$ higher with respect to the GM. This suggests that subsurface oxygen vacancies are not stable after water dissociation. The molecular adsorption at a $\mathrm{Ti}_{5 \mathrm{c}}$ site (Fig. 4e), which is second nearest-neighbour of $\mathrm{V}_{\mathrm{O}}^{\text {sub }}$, and at $\mathrm{Ti}_{4 \mathrm{c}}$ in the proximity of $\mathrm{V}_{\mathrm{O}}^{\text {surf }}$ (Fig. 4b) is, respectively, 0.59 and $0.61 \mathrm{eV}$ higher in energy with respect to the GM. The $\mathrm{Ti}_{5 \mathrm{c}}$ sites in the proximity of an oxygen vacancy are not equivalent. $\mathrm{MH}$ correctly found the $\mathrm{Ti}_{5 \mathrm{c}}$ adsorption site for the molecular adsorption which 
results in the lowest energy molecule/surface configuration: for $\mathrm{V}_{\mathrm{O}}^{\text {sub }}$ it is represented by the second nearest neighboring $\mathrm{Ti}_{5 \mathrm{c}}$ site (Ti-1 atom of Fig. 1), whilst for $\mathrm{V}_{\mathrm{O}}^{\text {surf }}$ it is the four-fold $\mathrm{Ti}_{4 \mathrm{c}}$ surface site. These preferential adsorption sites agree with previous investigations. ${ }^{55,60}$ Dissociation of $\mathrm{H}_{2} \mathrm{O}$ at $\mathrm{V}_{\mathrm{O}}^{\text {sub }}$ with hydrogen on the nearest-neighbour $\mathrm{O}_{3 \mathrm{c}}$ is $1.08 \mathrm{eV}$ higher in energy with respect to the GM (Fig. 4f). Considering the periodic boundary conditions on the surface plane xy, the distance between the oxygen atom of the $\mathrm{OH}$ group and $\mathrm{H}$ of Fig. $4 \mathrm{f}$ is $2.46 \AA$.

The order of low energy structures found by MH and their binding energies agrees with DFT and $a b$ initio molecular dynamics calculations of Tilocca et. al. ${ }^{60}$ They reported values of $-1.85 \mathrm{eV},-1.49 \mathrm{eV}$, and $-1.48 \mathrm{eV}$ for the GM (dissociative adsorption with two $\mathrm{OH}_{\mathrm{b}}$, Fig. 4a), the subsequent dissociative adsorption with one $\mathrm{OH}_{\mathrm{t}}$ and one $\mathrm{OH}_{\mathrm{b}}$ (Fig. $4 \mathrm{~d}$ ), and the molecular adsorption at $\mathrm{V}_{\mathrm{O}}^{\text {surf }}$ (Fig. 4b), respectively. Considering that $\mathrm{V}_{\mathrm{O}}^{\text {sub }}$ is energetically favoured at the $\mathrm{A}_{101}$ /vacuum interface, $\mathrm{H}_{2} \mathrm{O}$ first molecularly binds to the anatase (101) surface in the proximity of $\mathrm{V}_{\mathrm{O}}^{\text {sub }}$, and then dissociates, falling to its GM after crossing an energy barrier between the molecular and dissociated states. ${ }^{60}$ When a $\mathrm{V}_{\mathrm{O}}^{\text {surf }}$ is present, dissociative adsorption takes place. The opposite holds for $\mathrm{V}_{\mathrm{O}}^{\text {sub }}$ which favors molecular bonds. Combined STM and X-ray photoelectron spectroscopy analysis in UHV for defective nonstoichiometric $\mathrm{A}_{101}$ surface shows that molecular $\mathrm{H}_{2} \mathrm{O}$ preferentially adsorbs close to subsurface oxygen vacancy defects. ${ }^{55}$ Our structure predictions agree with this scenario since molecular adsorption on $\mathrm{V}_{\mathrm{O}}^{\text {sub }}$ is energetically favourable with respect to a dissociative interaction. Our results also agree with previous DFT calulations ${ }^{25}$ which employ a converged number of $\mathrm{O}-\mathrm{Ti}-\mathrm{O}$ layers, i.e. six trilayers, where $\mathrm{H}_{2} \mathrm{O}$ molecular adsorption on $\mathrm{V}_{\mathrm{O}}^{\text {sub }}$ is $0.12 \mathrm{eV}$ lower in energy with respect to molecular adsorption on $\mathrm{V}_{\mathrm{O}}^{\text {surf }}$.

The scenario is totally modified for $\mathrm{H}_{2} \mathrm{O}$ at the $\mathrm{A}_{101}$ /solvent interface, where the solvent is water. The GM is the same of the $\mathrm{A}_{101}$ /vacuum interface (Fig. 4a), but its binding energy drops to $-0.25 \mathrm{eV}$. The second lowest configuration in energy (0.22 eV from the GM) comes from the molecular binding of $\mathrm{H}_{2} \mathrm{O}$ at $\mathrm{V}_{\mathrm{O}}^{\text {surf }}$ (Fig. 4b). Its binding energy drops from a 
value of $-1.24 \mathrm{eV}$ in vacuum at $-0.033 \mathrm{eV}$ at the solid/liquid interface. The third lowest configuration in energy $\left(0.24 \mathrm{eV}\right.$ from GM, Fig. 4c) is represented by the $\mathrm{H}_{2} \mathrm{O}$ far away from the $A_{101}$ surface plunged in the bulk water, i.e. water leaves the surface. This suggests that water weakly interacts with the wet $\mathrm{A}_{101}$ defective surface, as already found for the perfect wet surface. However in presence of oxygen vacancy defects, water can adsorb although its binding energy is really small compared to the values in vacuum for the defective $A_{101}$. Structures near the GM arise from $\mathrm{H}_{2} \mathrm{O}$ dissociation at $\mathrm{V}_{\mathrm{O}}^{\text {surf }}$ (Fig. 4d), and subsequently molecular adsorption at $\mathrm{Ti}_{4 \mathrm{c}}$ sites, which are nearest-neighbosr of oxygen at vacancy defects (Fig. 4e). A binding energy of $-0.13 \mathrm{eV}$ is found for the molecular adsorption at a surface $\mathrm{Ti}_{5 \mathrm{c}}$, which is second nearest-neighbour of $\mathrm{V}_{\mathrm{O}}^{\text {sub }}$ (Fig. 4f). At the $\mathrm{A}_{101} /$ water interface with the presence of a surface oxygen vacancy $\mathrm{V}_{\mathrm{O}}^{\text {surf }}$, molecular and dissociative adsorption of $\mathrm{H}_{2} \mathrm{O}$ is almost isoenergetics. All binding energies are negative but less than $0.25 \mathrm{eV}$ in absolute value. Adsorption is thus more likely to occur on the defective than on the perfect $\mathrm{A}_{101}$ interface with water. Considering that surface vacancies $\mathrm{V}_{\mathrm{O}}^{\text {surf }}$ are more stable than at subsurface vacancies, surface defects play a central role on water adsorption.

$\mathrm{CH}_{3} \mathrm{CH}_{2} \mathrm{OH}$ shows dynamics similar to $\mathrm{H}_{2} \mathrm{O}$ at the $\mathrm{A}_{101} /$ vacuum interface. For both vacuum and wet interfaces, the global minimum is represented by a dissociated ethanol molecule, with the oxygen atom of the $\mathrm{CH}_{3} \mathrm{CH}_{2} \mathrm{O}$ ethoxide filling the $\mathrm{V}_{\mathrm{O}}^{\text {surf }}$ surface site $\left(\mathrm{EtO}_{\mathrm{b}}\right)$, and $\mathrm{H}$ binding to an $\mathrm{O}_{2 c}$ site, giving $\mathrm{OH}_{\mathrm{b}}$ (see Fig. 4g). Its binding energy is $-1.87 \mathrm{eV}$ at the $\mathrm{A}_{101} /$ vacuum and $-1.08 \mathrm{eV}$ at the $\mathrm{A}_{101} /$ solvent interface (the solvent is now ethanol described by the soft-sphere model). Differently from water, at the $\mathrm{A}_{101} /$ solvent interface ethanol is still strongly adsorbed. The dielectric constant of ethanol is 24.85 , lying in between the vacuum unity and the value of 78.36 for water. At the $\mathrm{A}_{101}$ /vacuum interface, the $\mathrm{CH}_{3} \mathrm{CH}_{2} \mathrm{OH}$ dissociative adsorption at $\mathrm{Ti}_{4 \mathrm{c}}$ sites which is nearest-neighbours of $\mathrm{V}_{\mathrm{O}}^{\text {surf }}$ (Fig. 4h) and $\mathrm{Ti}_{5 \mathrm{c}}$ which is second nearest-neighbours of $\mathrm{V}_{\mathrm{O}}^{\text {sub }}$ (Fig. 4i) is, respectively, 0.15 and $0.24 \mathrm{eV}$ higher in energy with respect to the GM. The second lowest energy structure represents a local minimum, crossing point before falling to the GM. The third configuration appears when 
ethanol dissociates to $\mathrm{Ti}_{5 \mathrm{c}}$ sites second nearest-neighbors of a $\mathrm{V}_{\mathrm{O}}^{\mathrm{sub}}$ (Ti-1 atom of Fig. 1), and the subsurface oxygen vacancy slightly moves up on the first trilayer $\left(V_{O}^{\text {inter }}\right.$, see subsurface oxygen movement at Fig. 4i). This suggests that the subsurface oxygen vacancies, as found for the dissociative adsorption of water, are not stable after ethanol dissociation. This local minimum is characterized by one $\mathrm{OH}_{\mathrm{b}}$, and the ethoxide adsorbed on top of a $\mathrm{Ti}_{5 \mathrm{c}}$ site $\left(\mathrm{EtO}_{\mathrm{Ti}}\right)$. Afterwards, $\mathrm{CH}_{3} \mathrm{CH}_{2} \mathrm{OH}$ molecular adsorption at $\mathrm{V}_{\mathrm{O}}^{\text {surf }}$ (Fig. $\left.4 \mathrm{j}\right)$ and $\mathrm{V}_{\mathrm{O}}^{\text {sub }}$ (Fig. $4 \mathrm{k}$ ) is, respectively, 0.59 and $0.64 \mathrm{eV}$ higher in energy with respect to the GM. The energy of an ethanol molecule far away from the $A_{101}$ surface, i.e. when it leaves the surface, is $1.49 \mathrm{eV}$ higher with the respect to the GM (Fig. 4l). Hansen et al. explored all adsorption mechanisms of ethanol on a rutile $\mathrm{TiO}_{2}$ (110) surface, ${ }^{26}$ including their interaction with oxygen vacancies. Although they are different polymorphs, our adsorption configurations at the $\mathrm{A}_{101}$ /vacuum interface agree with their STM measurements where ethanol adsorbs both molecularly and dissociatively. The STM movie they reported shows that molecularly adsorbed $\mathrm{EtOH}_{\mathrm{Ti}}$ diffuses along the [010] Ti trough (structure of Fig. 3b). Once it approaches a $\mathrm{V}_{\mathrm{O}}^{\text {surf }}$, it dissociates and falls in the GM represented by $\mathrm{EtO}_{\mathrm{b}}$ and $\mathrm{OH}_{\mathrm{b}}$ (Fig. 4g). The molecular adsorption at $\mathrm{V}_{\mathrm{O}}^{\text {surf }}$ is $0.59 \mathrm{eV}$ higher in energy with respect to the dissociative GM, where Hansen et al. reported a value of $0.55 \mathrm{eV}$ at the rutile $\mathrm{TiO}_{2}(110)$ surface. ${ }^{26}$

At the $\mathrm{A}_{101}$ / solvent interface, ethanol dissociation at $\mathrm{Ti}_{4 \mathrm{c}}$ sites nearest-neighbours of $\mathrm{V}_{\mathrm{O}}^{\text {surf }}$ (see Fig. 4h) and $\mathrm{V}_{\mathrm{O}}^{\text {sub }}$ (see Fig. 4i) are 0.15 and $0.32 \mathrm{eV}$ higher in energy with respect to the $\mathrm{GM}$, and energetically more stable than molecular adsorption both at $\mathrm{V}_{\mathrm{O}}^{\text {surf }}(0.48 \mathrm{eV}$ from GM, Fig. 4j) and at $\mathrm{V}_{\mathrm{O}}^{\text {sub }}(0.68 \mathrm{eV}$ from GM, Fig. 4k). At the solid/liquid interface ethanol binding energies are lower than at the $\mathrm{A}_{101}$ /vacuum interface, pointing out that the wet embedding weakens the interaction between solvent molecules and the anatase $\mathrm{TiO}_{2}$ (1 01 1) surface. However binding energies for various configurations are still negative, suggesting exothermic reactions where ethanol tends to passivate surface defects. The energy of an ethanol molecule far away from the $\mathrm{A}_{101}$ surface, i.e. plunged in bulk ethanol, is $1.09 \mathrm{eV}$ higher with the respect to the GM (Fig. 4l). 
In conclusion, water weakly interacts at the $\mathrm{A}_{101}$ /water interface, also in the presence of oxygen vacancy defects. It can adsorb or dissociatively passivate $\mathrm{V}_{\mathrm{O}}^{\text {surf }}$. The threedimensional hydrogen-bond network of water destabilizes such configurations, making binding energies small. A water molecule can form up to four hydrogen bonds with neighboring molecules since there are exactly the right numbers of positively-charged hydrogens and oxygen lone pairs so that every one of them can be involved in hydrogen bonding. ${ }^{61}$ However, ethanol has a hydrogen atom attached directly to an oxygen, and the latter still has two lone pairs as in a water molecule. Hydrogen bonding can occur between ethanol molecules, although not as effectively as in water. The hydrogen bonding is limited by the fact that there is only one hydrogen in each ethanol molecule with sufficient positive charge. The differences in hydrogen bonding rationalizes the higher boiling point of water with respect to ethanol.

At the $A_{101}$ /water interface with the presence of a surface oxygen vacancy $V_{\mathrm{O}}^{\text {surf }}$, molecular and dissociative adsorption of $\mathrm{H}_{2} \mathrm{O}$ is almost isoenergetics. Vacancy defects are expected to coexist with molecular $\mathrm{H}_{2} \mathrm{O}$. Hence, a water environment strongly destabilizes the first water layer and the underlying defect concentration. On the contrary, ethanol strongly adsorbs at the $\mathrm{A}_{101}$ surface both in vacuum and in wet environments, fully passivating oxygen vacancies $\mathrm{V}_{\mathrm{O}}^{\text {surf }}$ at $\mathrm{O}_{2 \mathrm{c}}$ bridges. Dissociative adsorption of ethanol is favorite with respect to molecular binding when a surface oxygen vacancy is present. Subsurface vacancies are destabilised after the dissociative adsorption of a water or ethanol molecule in the proximity of the defect site and start to migrate at surface sites. A further analysis of surface defects and their effects to photocatalytic properties can support our findings. The presence/absence of surface oxygen vacancies can be related to intragap shallow and deep electron/hole traps.

\subsection{Infrared and photoluminescence analysis}

To shed light on the interface of water and ethanol with the anatase $\mathrm{TiO}_{2}$ (101) surface, we analyzed anatase $\mathrm{TiO}_{2}$ nanoparticles by Fourier transform infrared spectroscopy, using a Perkin-Elmer Spectrum 1000 spectrometer, and by photoluminescence spectroscopy, using 
a Horiba Fluorolog Spectrofluorometer. Titania colloids were prepared by dispersing $3 \mathrm{mg}$ of anatase Titania (Sigma Aldrich, $<100 \mathrm{~nm}$ ) in $10 \mathrm{ml}$ of water or ethanol followed by ultrasound treatment for 15 minutes.

The anatase nanocrystals mainly expose two low index surfaces, i.e. (001) and (101), with the latter being the most stable. The (101) termination occupies more than $94 \%$ of the nanoparticle surface area. ${ }^{9}$ The relative energetics of the two surfaces induces a final polyhedric shape. We verified this polyhedric morphology of the titania nanoparticles by means of SEM after deposition on a Si substrate. The eventual co-presence of less stable minority surfaces, like the $(001)$ one, should not alter our experimental analysis since they are strongly reactive and, due to a fast passivation, they should not influence the scenario here discussed.

In order to discriminate between the contribution of "free" solvent molecules and chemior physi-sorbed molecules on titania, we have dropped the solution on a silicon substrate and acquired a spectrum in air after drying the solution.. Fig. 5 reports the FT-IR spectra of titania nanoparticles deposited on silicon substrate after being dispersed in deionized water or ethanol (blue and red curves, respectively). Absolute quantitative comparisons between the signals observed in the two samples are not possible, since the amount of titania deposited on the silicon substrates is not comparable for the two samples (i.e. ethanol and water). However, we can extract information about the interaction between the different solvents and the titania surface.

The transmittance bands in the region $400-1000 \mathrm{~cm}^{-1}$ are attributed to $\mathrm{Ti}-\mathrm{O}$ and/or Ti-O-Ti bonds. ${ }^{62}$ The broad band in the wavenumber region of $3000-3650 \mathrm{~cm}^{-1}$ and the peak at $1633 \mathrm{~cm}^{-1}$ are ascribed to $\mathrm{OH}$ stretching and $\mathrm{OH}$ bending, respectively. ${ }^{63}$ For the sample dispersed in ethanol and deposited on silicon substrate (red curve), we observe two evident signals at 2845 and $2923 \mathrm{~cm}^{-1}$ related to $\mathrm{CH}$ stretching, ${ }^{63,64}$ while $\mathrm{OH}$ related features seem to be not relevant. For comparison, we can consider the FT-IR spectrum of $\mathrm{TiO}_{2}$ nanoparticles in ethanol solvent (see Fig. S1 in the Supporting Information), where, beside 
the $\mathrm{CH}$ stretching mode, additional features are observed: $\mathrm{CO}$ stretching peaks between $1000-1200 \mathrm{~cm}^{-1},{ }^{63,64}$ peaks at $1431,1451,1637,1648$ and $1655 \mathrm{~cm}^{-1}$ assigned to CO and $\mathrm{OH}$ bending of the ethoxy species ${ }^{63,64} \mathrm{OH}$ stretching at $3000-3650 \mathrm{~cm}^{-1} .{ }^{63,64}$ We can deduce that ethanol still remains adsorbed on the titania surface after deposition on the substrate, as evidenced by the presence of $\mathrm{CH}$ signals. The vibrations related to $\mathrm{OH}$ and $\mathrm{CO}$ bonds are negligible in comparison with the spectrum acquired in ethanol and this suggests a strong interaction of ethanol on the titania surface by the oxygen atom, giving rise to a dissociation of ethanol molecules that remain bound to surface oxygen vacancies. In this way the $\mathrm{TiO}_{2}$ surface is passivated by the dissociated ethanol molecules that hinder the water vapour adsorption from the surrounding air environment in atmospheric conditions, as indicated by the lack of $\mathrm{OH}$ stretching and $\mathrm{OH}$ bending bands. These results are in agreement with our theoretical calculations, which report a binding energy of about $1 \mathrm{eV}$ for dissociated ethanol adsorbed on a surface oxygen vacancy (see Table 4 and Fig. 4).

For titania nanoparticles deposited on silicon substrate after being dispersed in deionized water the signals related to $\mathrm{OH}$ stretching and bending indicate the presence of $\mathrm{H}_{2} \mathrm{O}$ or $\mathrm{OH}$ adsorbed on the titania surface. It has to be noticed that due to the hydrophilicity of $\mathrm{TiO}_{2}$, the $\mathrm{H}_{2} \mathrm{O}$ and $\mathrm{OH}$ related features are observed also when untreated $\mathrm{TiO}_{2}$ is exposed to air (not shown), while after ethanol passivation $\mathrm{H}_{2} \mathrm{O}$ and $\mathrm{OH}$ related signals are not observed. These results agree with our DFT results for water adsorption at the $\mathrm{A}_{101}$ /vacuum interface, where water molecularly adsorbs at the ideal surface (see Table 3 and Fig. 3). When surface oxygen vacancies are present, water dissociates healing such surface defects.

The strong interaction in ethanol (which does not take place in water) as well as its power to passivate surface oxygen vacancies is ultimately demonstrated by our PL measurements in wet conditions.

Photoluminescence emission spectra are effective in tracking the behaviour of light-excited electrons and holes in semiconductors since PL emission is precisely the consequence of the recombination of free and trapped carriers. ${ }^{65-72}$ The spatial and energetic distribution of 


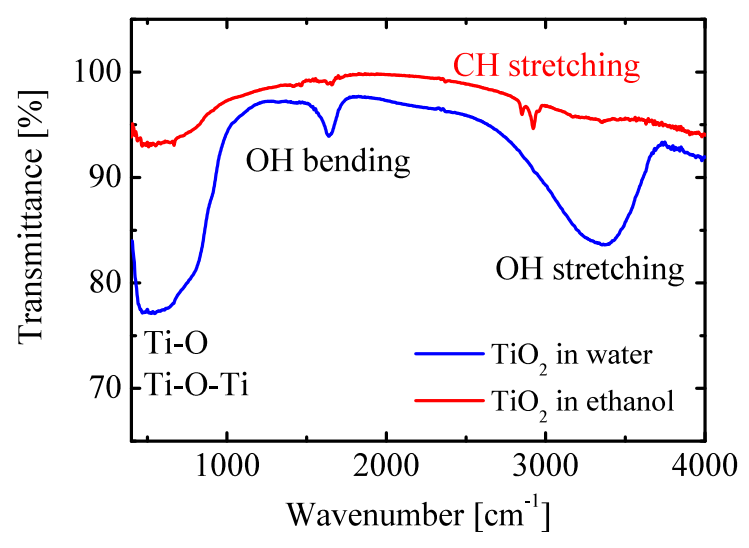

Figure 5: Fourier transform infrared spectra of titania nanoparticles deposited on silicon substrate after being dispersed in deionized water (blue curve) or ethanol (red curve).

intra-band states affects the performance of a photocatalyst by limiting recombination and carrier transport. ${ }^{68}$ In particular, shallow levels can favour the activation of electrons in the conduction band, their diffusion on the surface, as well as a longer $\mathrm{e}-\mathrm{h}$ recombination time, thus resulting in the case of photocatalysts like titania in an improvement of the photocatalytic activity. The contacting solvent can affect PL spectra influencing the carrier transport, by scavenging electrons or holes, by passivating surface defects which ultimately induce intragap trap states. ${ }^{68}$

Upon excitation with photon energies larger than the titania band gap, holes and electrons are formed on a time scale of the order of femtoseconds. Once produced, the charge carriers can be trapped into shallow and deep traps followed by their recombination. Earlier studies suggested that the anatase PL originates from radiative recombination of self-trapped exciton. ${ }^{73}$ However recent independent investigations correlate plotoluminescence spectra with radiative recombination processes involving surface and trap states. ${ }^{21,68,74}$ In particular, they suggest that the anatase PL spectra can be resolved in two physically distinct contributions, associated to two intraband gap traps: radiative recombination of conduction band and shallow trapped electrons with trapped holes (Type 1 or green PL, $\lambda \approx 510-530 \mathrm{~nm}$ - see schematic of Fig. 6, named G-PL) and radiative recombination of electrons in deep trap states with holes in the valence band (Type 2 or red PL, $\lambda \approx 650 \mathrm{~nm}$, named R-PL): ${ }^{21,68,74}$ 


$$
\begin{array}{ll}
e_{\mathrm{CB}}^{-}+h_{\mathrm{tr}}^{+} \longrightarrow h \nu_{\text {green }} & \text { (type } 1 \text { or green } \mathrm{PL}) \\
h_{\mathrm{VB}}^{+}+e_{\mathrm{tr}}^{-} \longrightarrow h \nu_{\text {red }} & \text { (type } 2 \text { or red } \mathrm{PL}) .
\end{array}
$$

Concerning the nature of such intraband traps, electrons localize in the interior of nanoparticles forming $\left(\mathrm{Ti}^{3+}\right)_{\text {latt }}{ }^{67}$ or undercoordinated surface titanium atoms ${ }^{21}$ (electron traps), while holes localize on surface oxygen vacancies ${ }^{21,59,74}$ (hole traps).

A wet environment is expected to strongly influence photoluminescence spectra, since the surface of the fully solvated nanoparticles will interact with solvent molecules, with a finite probability of charges transfer. Unsaturated titanium atoms are adsorption sites for $\mathrm{H}_{2} \mathrm{O}$ or $\mathrm{CH}_{3} \mathrm{CH}_{2} \mathrm{OH}$ (and their respective dissociation products, i.e. hydroxyl or ethoxide groups) through $\mathrm{Ti}-\mathrm{O}$ bonding, while undercoordinated oxygen atoms form hydrogen bonds $\left(\mathrm{O}-\mathrm{H}\right.$ bonds) with the water hydrogens. ${ }^{70}$ As a consequence, the red emission is quenched in both samples immersed in water or ethanol. Oxygen vacancies can induce water and ethanol dissociation, with the hydroxyl or ethoxide group filling the surface vacancy site. The schematic of Fig. 6 shows the electron photoexcitation from the valence to the conduction band, and the subsequent radiative recombination with a hole intragap trap (type 1 green PL). The intensity of this emission increases with increasing density of trap states and diminishes with increasing efficiency of carrier transport.

Fig. 6 reports photoluminescence spectra of anatase nanoparticles dispersed in water (blue curve) and ethanol (red curve), obtained with an excitation wavelength of $325 \mathrm{~nm}$ (3.81 eV) and using a $370 \mathrm{~nm}$ edge filter. The PL peak at about $400-420 \mathrm{~nm}$ is present both in water and ethanol, even if a wavelength shift is observed. As reported in previous studies, this peak is assigned to bulk self-trapped exciton, consistent with the contribution of $\mathrm{TiO}_{6}$ octahedra of anatase crystal structure. ${ }^{68}$

Our theoretical calculations reported in Table 3 and 4 predict weak molecular or disso- 
ciative bonds of $\mathrm{H}_{2} \mathrm{O}$ and $\mathrm{OH}$ to $\mathrm{Ti}_{5 c}$ at the $\mathrm{A}_{101}$ / water interface, and stronger interactions for molecular or dissociative ethanol. In both cases, the first solvation shell is predicted to complete the six-fold coordination of surface titanium atoms. The type $1 \mathrm{G}-\mathrm{PL}$ is present for anatase $\mathrm{TiO}_{2}$ nanoparticles dispersed in water $(\lambda \approx 534 \mathrm{~nm})$ and quenched when they are in ethanol solvent. This emission is associated with hole deep trap states due to surface oxygen vacancies. ${ }^{21,59,74}$ Considering the formation energies of Table 2, water favours oxygen vacancy defects at surface sites. The latter weakly interact with water molecules, and they are only partially healed. Table 4 reports the GM and low-lying metastable structures of $\mathrm{H}_{2} \mathrm{O}$ at the $\mathrm{A}_{101}$ /water interface. At the $\mathrm{A}_{101}$ /water interface with the presence of a surface oxygen vacancy $\mathrm{V}_{\mathrm{O}}^{\text {surf }}$, molecular and dissociative adsorption of $\mathrm{H}_{2} \mathrm{O}$ is almost isoenergetics. Water weakly interacts with the $\mathrm{A}_{101}$ defective surface with binding energies less than 0.25 $\mathrm{eV}$. Such small interaction suggests that at equilibrium surface vacancies $\mathrm{V}_{\mathrm{O}}^{\text {surf }}$ co-exist with healed vacancy defects. As a consequence, they can introduce depth hole intragap traps, as evinced by PL spectroscopy in wet conditions.

On the contrary, ethanol strongly interacts at $\mathrm{A}_{101}$ /ethanol interface. Binding energies of GM and low energy configurations of Table 4 are on the order of $1.00 \mathrm{eV}$. After its dissociation, ethanol fully passivates surface oxygen vacancies in wet conditions. Depth hole trap states are healed and its corresponding PL signal is quenched. As a consequence, the PL spectra are fully consistent with our DFT structure prediction calculations both in water and ethanol.

Mercado and co-workers investigated the location of hole and electron traps on specific facets of anatase nanocrystals. ${ }^{21}$ They reported PL measurements of sintered and unsintered films of $\mathrm{TiO}_{2}$ nanoparticles (which expose predominantly (1 $\left.\begin{array}{lll}1 & 1\end{array}\right)$ surfaces), nanosheets (exposing predominantly (0 01 ) surfaces) and nanotubes (with (110) and (100) facets). Pallotti and co-workers investigated PL and excitation-resolved PL of nano-structured and quasionedimensional $\mathrm{TiO}_{2}$ films deposited on silicon substrates by pulsed laser deposition(PLD). ${ }^{74}$ Our results fully agree with their PL assignments of green hole trap emission (0.7-1.4 eV 
above the valence band) to oxygen vacancies on (1 011$)$ surfaces.

The enhancement of the green emission of anatase on vacuum annealing also suggests that this PL is associated with oxygen vacancies, ${ }^{75}$ supporting our picture where ethanol fully passivates surface vacancy defects on (101) surfaces, quenching the green PL signal.

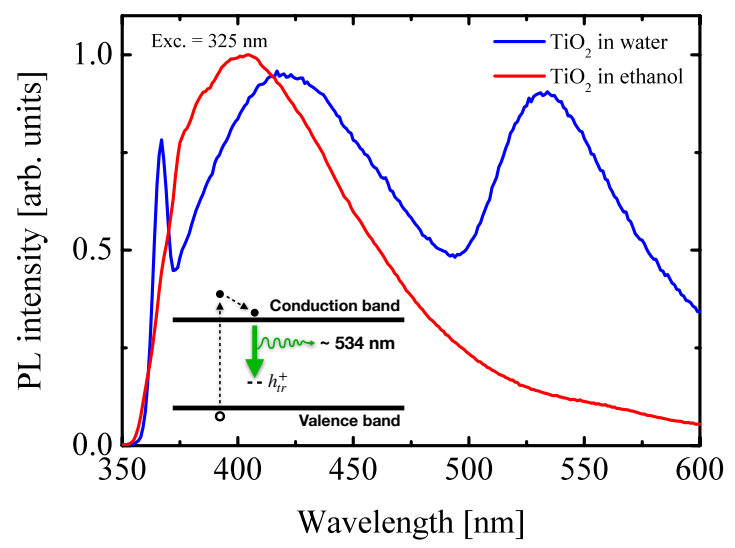

Figure 6: Photoluminescence spectra of anatase nanoparticles dispersed in water (blue curve) and ethanol (red curve), obtained with an excitation wavelength of $325 \mathrm{~nm}$. The schematic reports the electron photoexcitation from the valence to the conduction band, and the subsequent radiative recombination with a hole intragap trap (type 1 , green PL).

\section{Conclusions}

We investigated the interface of anatase $\mathrm{TiO}_{2}\left(\begin{array}{lll}1 & 0 & 1\end{array}\right)$ surface with water and ethanol solvents.

Methodology. We showed that the inclusion of the wet environment in the methodological scheme is fundamental for a proper exploration of the potential energy surfaces of wet systems and solid/liquid interfaces. The structural stability as well as the energetic order of local minima are strongly dependent on the surrounding solvent. The calculations utilized minima hopping structure predictions at a density functional theory level for single molecules on the $\mathrm{TiO}_{2}$ (101) surface under vacuum and wet conditions. The latter were modelled by means of a soft-sphere implicit solvation model. We validated the computational paradigm reproducing surface atomic displacements extracted from previous experimental surface X- 
ray diffraction measurements and DFT calculations with explicit solvent molecules.

Water and ethanol adsorption. We computed the formation energy of oxygen vacancy defects lying at surface sites in contact with water and ethanol. We found that the wet environment at the solid/liquid interface modifies the relative stability of surface and subsurface oxygen vacancies with respect to the interface with vacuum. Subsurface oxygen vacancies are more stable than surface ones at the vacuum interface. Surface and subsurface vacancy sites are almost isoenergetic at the $\mathrm{A}_{101}$ /ethanol interface, whilst surface oxygen vacancies are energetically favorable than subsurface ones at the $\mathrm{A}_{101}$ / water interface. Vacancy energetics is confirmed by ab initio molecular dynamics simulations with explicit water molecules. A subsurface oxygen vacancy moves spontaneously towards a superficial position during MD kinetics of a few ps, showing that the barrier between the two configurations is very low. Vacancy energetics is also in agreement with structure predictions at the solid/liquid interface for the molecular adsorption. The dissociative adsorption of water and ethanol molecules in the proximity of a subsurface vacancy destabilizes the latter and promotes its diffusion to surface sites. In the proximity of a surface vacancy, dissociative adsorption is favorite with respect to molecular one for ethanol whilst it is almost isoenergetic for a water molecule. Water molecules only weakly interact with the wet $\mathrm{A}_{101}$ termination, and surface oxygen vacancy defects coexist with adsorbed molecules. Ethanol molecules strongly passivate surface oxygen vacancies.

The predicted structures correctly rationalize the experimental infrared and photolumiscence measurements of anatase nanoparticles exposing predominantly (10 1) surfaces dispersed in water and ethanol solvents. We report transmittance bands of Fourier transform infrared spectra of titania nanoparticles deposited on a silicon substrate, after being dispersed in deionized water or ethanol. IR spectra agrees with our DFT results for water adsorption at the $\mathrm{A}_{101}$ /vacuum interface, where water molecularly adsorbs at the ideal surface. On the other side, at the solid/liquid interfaces ethanol dissociates and strongly adsorbs at surface vacancy sites. Photoluminescence spectra revealed that the type 2 red PL emission 
is quenched both in water and ethanol. Being related to deep electron traps induced by undercoordinated surface titanium atoms, it agrees with the molecular adsorption mechanism found by the structure predictions. Both water and ethanol adsorb at undercoordinated surface titanium atoms forming $\mathrm{O}-\mathrm{Ti}$ bonds. The type 1 green PL is present for anatase $\mathrm{TiO}_{2}$ nanoparticles dispersed in water $(\lambda \approx 534 \mathrm{~nm})$ and quenched when they are in the ethanol solvent. Green PL is associated with the radiative recombination of conduction band and shallow trapped electrons with trapped holes, located at surface oxygen vacancies. This result agrees with our simulations where water weakly binds to the $A_{101}$ defective surface with DFT binding energies less than $0.25 \mathrm{eV}$. Such small interaction suggests that at equilib-

rium surface vacancies $\mathrm{V}_{\mathrm{O}}^{\text {surf }}$ co-exist with healed vacancy defects. On the contrary, ethanol strongly interacts at the $\mathrm{A}_{101}$ / ethanol interface. DFT binding energies of GM and low energy configurations are on the order of $1 \mathrm{eV}$. After its dissociation, ethanol fully passivates surface oxygen vacancies in wet conditions. Depth hole trap states are healed and their corresponding PL signal is quenched. The match of structure predictions with implicit solvation and experimental IR and PL measurements, validate the whole computational approach.

\section{Acknowledgement}

S.G. acknowledges the support from the Swiss National Science Foundation and the NCCR MARVEL. Computer resources were provided by the Swiss National Supercomputing Centre (CSCS) under Project ID s869.

\section{Supporting Information Available}

The following files are available free of charge.

- Supporting Information: Fig. S1 which reports the infrared spectra of Titania nanoparticles dispersed in ethanol solvent; 
- A movie TiO2-H2O-interface-Ovacancy-migration.mp4 showing the molecular dynamics simulation for the migration of a subsurface oxygen vacancy towards a surface site at the Anatase $\mathrm{TiO}_{2}$ (101) interface with explicit water molecules;

- All the optimized structures used for the calculations (file all-structures-Anatase-1014x1-6-TiO2-trilayers.zip):

- Perfect anatase $\mathrm{TiO}_{2}$ (101) surface modeled with six trilayers of a $4 \times 1$ supercell (288 atoms in total) used for Table 1 and 2;

- Anatase $\mathrm{TiO}_{2}$ (10 1) surface with an oxygen surface/subsurface vacancy used for the energetics of Table 2;

- Structures for water/ethanol adsorption used for Table 3 and Figure 3;

- Structures for water/ethanol adsorption used for Table 4 and Figure 4.

\section{References}

(1) Chen, X.; Mao, S. S. Titanium Dioxide Nanomaterials: Synthesis, Properties, Modifications, and Applications. Chem. Rev. 2007, 107, 2891-2959.

(2) Fujishima, A.; Honda, K. Electrochemical Photolysis of Water at a Semiconductor Electrode. Nature 1972, 238, 37-38.

(3) Asahi, R.; Morikawa, T.; Ohwaki, T.; Aoki, K.; Taga, Y. Visible-Light Photocatalysis in Nitrogen-Doped Titanium Oxides. Science 2001, 293, 269-271.

(4) Filice, S.; Compagnini, G.; Fiorenza, R.; Scirè, S.; D’Urso, L.; Fragalà, M. E.; Russo, P.; Fazio, E.; Scalese, S. Laser Processing of $\mathrm{TiO}_{2}$ Colloids for an Enhanced Photocatalytic Water Splitting Activity. J. Colloid Interface Sci. 2017, 489, 131 - 137.

(5) Filice, S.; D'Angelo, D.; Scarangella, A.; Iannazzo, D.; Compagnini, G.; Scalese, S. 
Highly Effective and Reusable Sulfonated Pentablock Copolymer Nanocomposites for Water Purification Applications. RSC Adv. 2017, 7, 45521-45534.

(6) Grätzel, M. Photoelectrochemical Cells. Nature 2001, 414, 338-344.

(7) Daghrir, R.; Drogui, P.; Robert, D. Modified $\mathrm{TiO}_{2}$ For Environmental Photocatalytic Applications: A Review. Ind. Eng. Chem. Res. 2013, 52, 3581-3599.

(8) Ranade, M. R.; Navrotsky, A.; Zhang, H. Z.; Banfield, J. F.; Elder, S. H.; Zaban, A.; Borse, P. H.; Kulkarni, S. K.; Doran, G. S.; Whitfield, H. J. Energetics of Nanocrystalline $\mathrm{TiO}_{2}$. Proc. Natl. Acad. Sci. 2002, 99, 6476-6481.

(9) Lazzeri, M.; Vittadini, A.; Selloni, A. Structure and Energetics of Stoichiometric $\mathrm{TiO}_{2}$ Anatase Surfaces. Phys. Rev. B 2001, 63, 155409.

(10) He, Y.; Tilocca, A.; Dulub, O.; Selloni, A.; Diebold, U. Local Ordering and Electronic Signatures of Submonolayer Water on Anatase $\mathrm{TiO}_{2}(101)$. Nat. Mater. 2009, 8, 585 .

(11) Hussain, H.; Tocci, G.; Woolcot, T.; Torrelles, X.; Pang, C. L.; Humphrey, D. S.; Yim, C. M.; Grinter, D. C.; Cabailh, G.; Bikondoa, O.; et al, Structure of a Model $\mathrm{TiO}_{2}$ Photocatalytic Interface. Nat. Mater. 2016, 16, 461.

(12) Puga, A. V.; Forneli, A.; García, H.; Corma, A. Production of $\mathrm{H}_{2}$ by Ethanol Photoreforming on $\mathrm{Au} / \mathrm{TiO}_{2}$. Adv. Funct. Mater. 2014, 24, 241-248.

(13) Idriss, H.; Seebauer, E. Reactions of Ethanol over Metal Oxides. J. Mol. Catal. A: Chem. 2000, 152, $201-212$.

(14) Walle, L. E.; Borg, A.; Johansson, E. M. J.; Plogmaker, S.; Rensmo, H.; Uvdal, P.; Sandell, A. Mixed Dissociative and Molecular Water Adsorption on Anatase TiO2(101). J. Phys. Chem. C 2011, 115, 9545-9550.

(15) Patrick, C. E.; Giustino, F. Structure of a Water Monolayer on the Anatase $\mathrm{TiO}_{2}(101)$ Surface. Phys. Rev. Applied 2014, 2, 014001. 
(16) Nadeem, I. M.; Treacy, J. P. W.; Selcuk, S.; Torrelles, X.; Hussain, H.; Wilson, A.; Grinter, D. C.; Cabailh, G.; Bikondoa, O.; Nicklin, C.; et al, Water Dissociates at the Aqueous Interface with Reduced Anatase $\mathrm{TiO}_{2}$ (101). J. Phys. Chem. Lett. 2018, 9, $3131-3136$.

(17) Martinez-Casado, R.; Mallia, G.; Harrison, N. M.; PÃl'rez, R. First-Principles Study of the Water Adsorption on Anatase(101) as a Function of the Coverage. J. Phys. Chem. C 2018, 122, 20736-20744.

(18) Calegari Andrade, M. F.; Ko, H.-Y.; Car, R.; Selloni, A. Structure, Polarization, and Sum Frequency Generation Spectrum of Interfacial Water on Anatase TiO2. J. Phys. Chem. Lett. 2018, 9, 6716-6721.

(19) He, Y.; Dulub, O.; Cheng, H.; Selloni, A.; Diebold, U. Evidence for the Predominance of Subsurface Defects on Reduced Anatase $\mathrm{TiO}_{2}(101)$. Phys. Rev. Lett. 2009, 102, 106105.

(20) Cheng, H.; Selloni, A. Surface and Subsurface Oxygen Vacancies in Anatase $\mathrm{TiO}_{2}$ and Differences with Rutile. Phys. Rev. B 2009, 79, 092101.

(21) Mercado, C. C.; Knorr, F. J.; McHale, J. L.; Usmani, S. M.; Ichimura, A. S.; Saraf, L. V. Location of Hole and Electron Traps on Nanocrystalline Anatase $\mathrm{TiO}_{2}$. J. Phys. Chem. C 2012, 116, 10796-10804.

(22) Ali, H.; Seidel, R.; Bergmann, A.; Winter, B. Electronic Structure of Aqueous-Phase Anatase Titanium Dioxide Nanoparticles Probed by Liquid Jet Photoelectron Spectroscopy. J. Mater. Chem. A 2019, 7, 6665-6675.

(23) Marzari, N. The Frontiers and the Challenges. Nat. Mater. 2016, 15, 381.

(24) Herman, G. S.; Dohnálek, Z.; Ruzycki, N.; Diebold, U. Experimental Investigation of 
the Interaction of Water and Methanol with Anatase- $\mathrm{TiO}_{2}(101)$. J. Phys. Chem. B 2003, 107, 2788-2795.

(25) Fan, J.; Zhao, J. Z.; Xu, H.; Tong, S. Y. Comment on "Interplay between Water and $\mathrm{TiO}_{2}$ Anatase (101) Surface with Subsurface Oxygen Vacancy". Phys. Rev. Lett. 2015, $115,149601$.

(26) Hansen, J. O.; Huo, P.; Martinez, U.; Lira, E.; Wei, Y. Y.; Streber, R.; Lægsgaard, E.; Hammer, B.; Wendt, S.; Besenbacher, F. Direct Evidence for Ethanol Dissociation on Rutile $\mathrm{TiO}_{2}(110)$. Phys. Rev. Lett. 2011, 10\%, 136102.

(27) Katsiev, K.; Harrison, G.; Alghamdi, H.; Alsalik, Y.; Wilson, A.; Thornton, G.; Idriss, H. Mechanism of Ethanol Photooxidation on Single-Crystal Anatase $\mathrm{TiO}_{2}(101)$. J. Phys. Chem. C 2017, 121, 2940-2950.

(28) Sprik, M.; Hutter, J.; Parrinello, M. Ab Initio Molecular Dynamics Simulation of Liquid Water: Comparison of Three Gradient-Corrected Density functionals. J. Chem. Phys. 1996, 105, 1142-1152.

(29) Grossman, J. C.; Schwegler, E.; Draeger, E. W.; Gygi, F.; Galli, G. Towards an Assessment of the Accuracy of Density Functional Theory for First Principles Simulations of Water. J. Chem. Phys. 2004, 120, 300-311.

(30) VandeVondele, J.; Mohamed, F.; Krack, M.; Hutter, J.; Sprik, M.; Parrinello, M. The Influence of Temperature and Density Functional Models in Ab Initio Molecular Dynamics Simulation of Liquid Water. J. Chem. Phys. 2005, 122, 014515.

(31) Sit, P. H.-L.; Marzari, N. Static and Dynamical Properties of Heavy Water at Ambient Conditions from First-Principles Molecular Dynamics. J. Chem. Phys. 2005, 122, 204510. 
(32) Fisicaro, G.; Genovese, L.; Andreussi, O.; Marzari, N.; Goedecker, S. A Generalized Poisson and Poisson-Boltzmann Solver for Electrostatic Environments. J. Chem. Phys. 2016, $144,014103$.

(33) Fisicaro, G.; Genovese, L.; Andreussi, O.; Mandal, S.; Nair, N. N.; Marzari, N.; Goedecker, S. Soft-Sphere Continuum Solvation in Electronic-Structure Calculations. J. Chem. Theory Comput. 2017, 13, 3829-3845.

(34) Andreussi, O.; Fisicaro, G. Continuum Embeddings in Condensed-Matter Simulations. Int. J. Quantum Chem. 2019, 119, e25725.

(35) Goedecker, S. Minima Hopping: An Efficient Eearch Method for the Global Minimum of the Potential Energy Surface of Complex Molecular Systems. J. Chem. Phys. 2004, 120, 9911-9917.

(36) Genovese, L.; Neelov, A.; Goedecker, S.; Deutsch, T.; Ghasemi, S. A.; Willand, A.; Caliste, D.; Zilberberg, O.; Rayson, M.; Bergman, A.; et al, Daubechies Wavelets as a Basis Set for Density Functional Pseudopotential Calculations. J. Chem. Phys. 2008, 129, 014109.

(37) http://www.bigdft.org

(38) Goedecker, S.; Teter, M.; Hutter, J. Separable Dual-Space Gaussian Pseudopotentials. Phys. Rev. B 1996, 54, 1703-1710.

(39) Willand, A.; Kvashnin, Y. O.; Genovese, L.; Vázquez-Mayagoitia, A.; Deb, A. K.; Sadeghi, A.; Deutsch, T.; Goedecker, S. Norm-Conserving Pseudopotentials with Chemical Accuracy Compared to All-Electron Calculations. J. Chem. Phys. 2013, 138, 104109.

(40) Marques, M. A.; Oliveira, M. J.; Burnus, T. Libxc: A Library of Exchange and Cor- 
relation Functionals for Density Functional Theory. Comput. Phys. Comm. 2012, 183, $2272-2281$.

(41) Fisicaro, G.; Sicher, M.; Amsler, M.; Saha, S.; Genovese, L.; Goedecker, S. Surface Reconstruction of Fluorites in Vacuum and Aqueous Environment. Phys. Rev. Materials 2017, 1, 033609.

(42) Puglisi, R. A.; Caccamo, S.; Bongiorno, C.; Fisicaro, G.; Genovese, L.; Goedecker, S.; Mannino, G.; La Magna, A. Direct Observation of Single Organic Molecules Grafted on the Surface of a Silicon Nanowire. Sci. Rep. 2019, 9, 5647.

(43) Reddy, K. M.; Manorama, S. V.; Reddy, A. R. Bandgap Studies on Anatase Titanium Dioxide Nanoparticles. Mater. Chem. Phys. 2003, 78, $239-245$.

(44) Hebenstreit, W.; Ruzycki, N.; Herman, G. S.; Gao, Y.; Diebold, U. Scanning Tunneling Microscopy Investigation of the $\mathrm{TiO}_{2}$ Anatase (101) Surface. Phys. Rev. B 2000, 62, R16334-R16336.

(45) Treacy, J. P. W.; Hussain, H.; Torrelles, X.; Grinter, D. C.; Cabailh, G.; Bikondoa, O.; Nicklin, C.; Selcuk, S.; Selloni, A.; Lindsay, R.; et al, Geometric Structure of Anatase $\mathrm{TiO}_{2}(101)$. Phys. Rev. B 2017, 95, 075416.

(46) Labat, F.; Baranek, P.; Adamo, C. Structural and Electronic Properties of Selected Rutile and Anatase $\mathrm{TiO}_{2}$ Surfaces: An ab Initio Investigation. J. Chem. Theory Comput. 2008, 4, 341-352.

(47) Tomasi, J.; Mennucci, B.; Cammi, R. Quantum Mechanical Continuum Solvation Models. Chem. Rev. 2005, 105, 2999-3094.

(48) Andreussi, O.; Dabo, I.; Marzari, N. Revised self-consistent continuum solvation in electronic-structure calculations. J. Chem. Phys. 2012, 136, 064102. 
(49) Buckley, F.; Maryott, A. A. Tables of Dielectric Dispersion Data for Pure Liquids and Dilute Solutions; U.S. Dept. of Comerce, National Bureau of Standards, 1958; pp 6-8.

(50) Vittadini, A.; Selloni, A.; Rotzinger, F. P.; Grätzel, M. Structure and Energetics of Water Adsorbed at $\mathrm{TiO}_{2}$ Anatase (101) and (001) Surfaces. Phys. Rev. Lett. 1998, 81, 2954-2957.

(51) Scheiber, P.; Fidler, M.; Dulub, O.; Schmid, M.; Diebold, U.; Hou, W.; Aschauer, U.; Selloni, A. (Sub)Surface Mobility of Oxygen Vacancies at the $\mathrm{TiO}_{2}$ Anatase (101) Surface. Phys. Rev. Lett. 2012, 109, 136103.

(52) Lazzeri, M.; Vittadini, A.; Selloni, A. Erratum: Structure and Energetics of Stoichiometric $\mathrm{TiO}_{2}$ Anatase Surfaces [Phys. Rev. B 63, 155409 (2001)]. Phys. Rev. B 2002, $65,119901$.

(53) Li, H.; Guo, Y.; Robertson, J. Calculation of $\mathrm{TiO}_{2}$ Surface and Subsurface Oxygen Vacancy by the Screened Exchange Functional. J. Phys. Chem. C 2015, 119, 1816018166.

(54) Jensen, F. Introduction to Computational Chemistry, 3rd Edition; John Wiley \& Sons Ltd, 2017; pp 1-660.

(55) Aschauer, U.; He, Y.; Cheng, H.; Li, S.-C.; Diebold, U.; Selloni, A. Influence of Subsurface Defects on the Surface Reactivity of $\mathrm{TiO}_{2}$ : Water on Anatase (101). J. Phys. Chem. C 2010, 114, 1278-1284.

(56) Zhang, R.; Liu, Z.; Ling, L.; Wang, B. The Effect of Anatase $\mathrm{TiO}_{2}$ Surface Structure on the Behavior of Ethanol Adsorption and its Initial Dissociation Step: A DFT Study. Appl. Surf. Sci. 2015, 353, $150-157$.

(57) Setvin, M.; Daniel, B.; Aschauer, U.; Hou, W.; Li, Y.-F.; Schmid, M.; Selloni, A.; 
Diebold, U. Identification of Adsorbed Molecules via STM Tip Manipulation: $\mathrm{CO}, \mathrm{H}_{2} \mathrm{O}$, and $\mathrm{O}_{2}$ on $\mathrm{TiO}_{2}$ Anatase (101). Phys. Chem. Chem. Phys. 2014, 16, 21524-21530.

(58) Xu, C.; Yang, W.; Guo, Q.; Dai, D.; Chen, M.; Yang, X. Molecular Hydrogen Formation from Photocatalysis of Methanol on Anatase- $\mathrm{TiO}_{2}(101)$. J. Am. Chem. Soc. 2014, 136, $602-605$.

(59) Martínez-Casado, R.; Todorović, M.; Mallia, G.; Harrison, N. M.; Pérez, R. First Principles Calculations on the Stoichiometric and Defective (101) Anatase Surface and Upon Hydrogen and H2Pc Adsorption: The Influence of Electronic Exchange and Correlation and of Basis Set Approximations. Front. Chem. 2019, \%, 220.

(60) Tilocca, A.; Selloni, A. Reaction Pathway and Free Energy Barrier for Defect-Induced Water Dissociation on the (101) Surface of $\mathrm{TiO}_{2}$-Anatase. J. Chem. Phys. 2003, 119, $7445-7450$.

(61) DS, E.; WK, K. The Structure and Properties of Water; Oxford University Press, 2005, 2007; pp 1-320.

(62) Hosseini, M. M.; Kolvari, E.; Zolfagharinia, S.; Hamzeh, M. Simple Hydrothermal Synthesis of Sphere-Like $\mathrm{TiO}_{2}$ Nanoparticles and Their Functionalization with 1,4-Butane Sultone as a New Heterogeneous Catalyst. J. Iran. Chem. Soc. 2017, 14, 1777-1788.

(63) Rintramee, K.; Föttinger, K.; Rupprechter, G.; Wittayakun, J. Ethanol Adsorption and Oxidation on Bimetallic Catalysts Containing Platinum and Base Metal Oxide Supported on MCM-41. Appl. Catal. B-Environ. 2012, 115-116, 225 - 235.

(64) Llorca, J.; Homs, N.; de la Piscina, P. R. In Situ DRIFT-Mass Spectrometry Study of the Ethanol Steam-Reforming Reaction over Carbonyl-Derived Co/ZnO Catalysts. J. Catal. 2004, 227, $556-560$. 
(65) Jung, K. Y.; Park, S. B.; Anpo, M. Photoluminescence and Photoactivity of Titania Particles Prepared by the Sol-Gel Technique: Effect of Calcination Temperature. $J$. Photochem. Photobiol. A-Chem. 2005, 170, $247-252$.

(66) Gfroerer, T. H. Encyclopedia of Analytical Chemistry; American Cancer Society, 2006.

(67) Dimitrijevic, N. M.; Saponjic, Z. V.; Rabatic, B. M.; Poluektov, O. G.; Rajh, T. Effect of Size and Shape of Nanocrystalline $\mathrm{TiO}_{2}$ on Photogenerated Charges. An EPR Study. J. Phys. Chem. C 2007, 111, 14597-14601.

(68) Knorr, F. J.; Mercado, C. C.; McHale, J. L. Trap-State Distributions and Carrier Transport in Pure and Mixed-Phase $\mathrm{TiO}_{2}$ : Influence of Contacting Solvent and Interphasial Electron Transfer. J. Phys. Chem. C 2008, 112, 12786-12794.

(69) Mattioli, G.; Filippone, F.; Alippi, P.; Amore Bonapasta, A. Ab Initio Study of the Electronic States Induced by Oxygen Vacancies in Rutile and Anatase $\mathrm{TiO}_{2}$. Phys. Rev. B 2008, 78, 241201.

(70) Sun, C.; Liu, L.-M.; Selloni, A.; Lu, G. Q. M.; Smith, S. C. Titania-Water Interactions: a Review of Theoretical Studies. J. Mater. Chem. 2010, 20, 10319-10334.

(71) Buha, J. Photoluminescence Study of Carbon Doped and Hydrogen Co-Doped TiO2 Thin Films. Thin Solid Films 2013, 545, $234-240$.

(72) Grigioni, I.; Dozzi, M. V.; Bernareggi, M.; Chiarello, G. L.; Selli, E. Photocatalytic CO2 Reduction vs. H2 Production: The Effects of Surface Carbon-Containing Impurities on the Performance of TiO2-Based Photocatalysts. Catal. Today 2017, 281, $214-220$, Heterogeneous Photocatalyis from fundamentals to possible applications.

(73) Tang, H.; Berger, H.; Schmid, P.; Lévy, F.; Burri, G. Photoluminescence in $\mathrm{TiO}_{2}$ Anatase Single Crystals. Solid State Commun. 1993, 87, 847-850. 
(74) Pallotti, D. K.; Passoni, L.; Maddalena, P.; Di Fonzo, F.; Lettieri, S. Photoluminescence Mechanisms in Anatase and Rutile $\mathrm{TiO}_{2}$. J. Phys. Chem. C 2017, 121, 9011-9021.

(75) McHale, J. L.; Rich, C. C.; Knorr, F. J. Trap State Photoluminescence of Nanocrystalline and Bulk $\mathrm{TiO}_{2}$ : Implications for Carrier Transport. MRS Proceedings 2010, 1268, EE03-08.

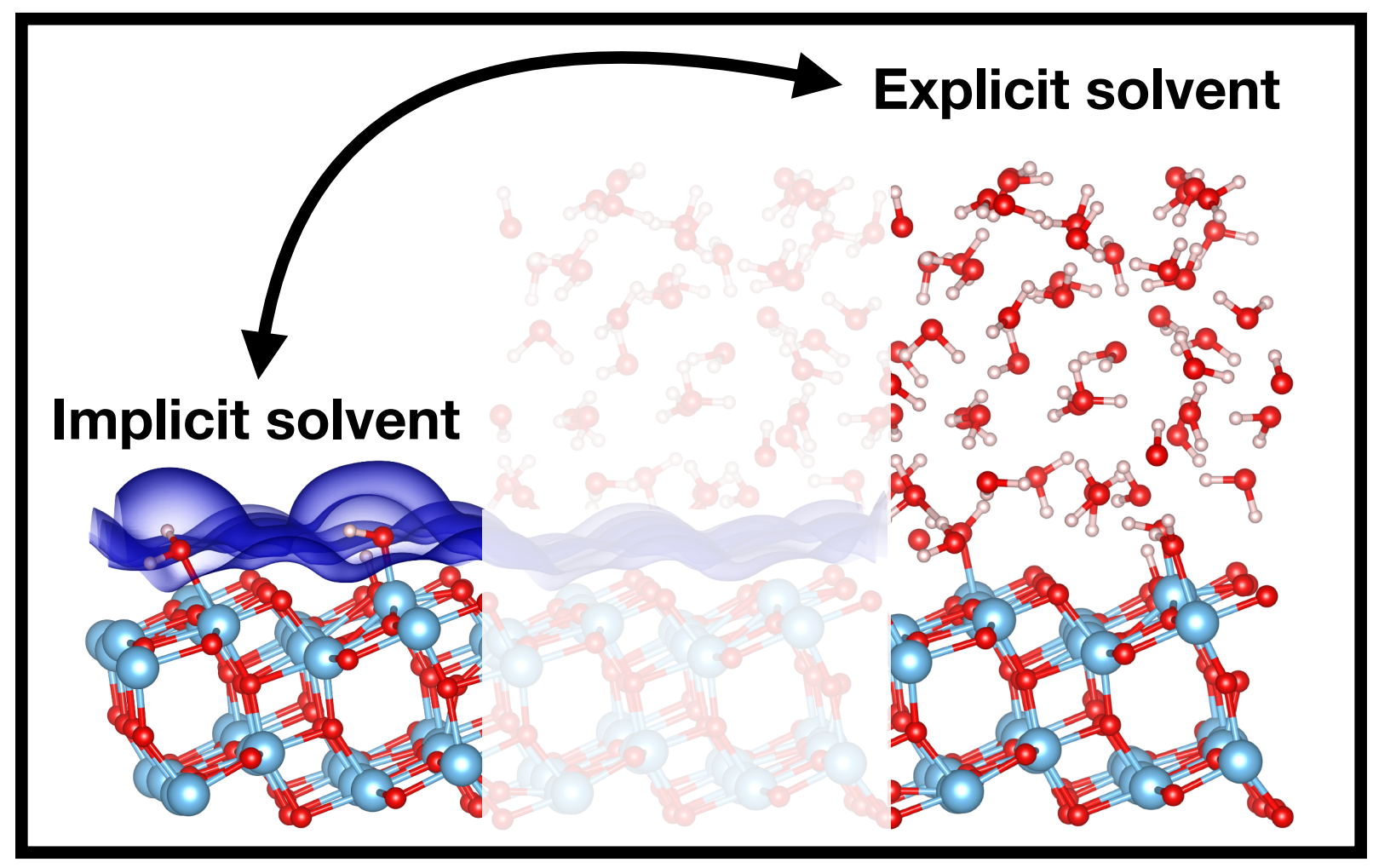

TOC graphic: For Table of Contents Only. 